\title{
Lot Sizing with Inventory Bounds and Fixed Costs: Polyhedral Study and Computation
}

\author{
Alper Atamtürk, Simge Küçükyavuz \\ Department of Industrial Engineering and Operations Research, University of California, Berkeley, California 94720-1777 \\ \{atamturk@ieor.berkeley.edu, simge@ieor.berkeley.edu\}
}

\begin{abstract}
We investigate the polyhedral structure of the lot-sizing problem with inventory bounds. We consider two models, one with linear cost on inventory, the other with linear and fixed costs on inventory. For both models, we identify facetdefining inequalities that make use of the inventory bounds explicitly and give exact separation algorithms. We also describe a linear programming formulation of the problem when the order and inventory costs satisfy the Wagner-Whitin nonspeculative property. We present computational experiments that show the effectiveness of the results in tightening the linear programming relaxations of the lot-sizing problem with inventory bounds and fixed costs.
\end{abstract}

Subject classifications: lot sizing; facets; separation algorithms; computation.

Area of review: Optimization.

History: Received July 2003; revision received February 2004; accepted July 2004.

\section{Introduction}

Given the demand for an item for each time period over a finite discrete horizon, the lot-sizing problem is to determine the order and inventory quantity in each time period so that the sum of order and inventory holding costs is minimized. In this paper, we study the problem with linear and fixed costs on order as well as on inventory. Furthermore, we impose upper bounds on the inventory carried in each period. We refer to this problem as the lot-sizing problem with bounded inventory. Throughout we assume that there is no upper bound on the order quantity.

\section{Motivation}

Even though in almost every study on the lot-sizing problem inventory is assumed to be unbounded, for many practical applications the amount of inventory that is carried from one period to the next is bounded due to either physical constraints such as warehouse capacity or managerial policies.

Another aspect of the problem studied here, which has not received much attention in the literature, is the inventory fixed costs. Inventory fixed costs and capacities play a significant role in situations where warehousing is outsourced. The lease cost of storage space in an off-site warehouse is a fixed charge that cannot be treated as part of the standard variable holding cost, which is commonly taken as the cost of capital investment in inventory. Inventory fixed costs and capacities also arise in situations where manufacturers rent shelf space at the retailers. These fixed rental costs and limited storage capacities are considerations that cannot be overlooked.

It has been demonstrated in earlier studies (Pochet and Wolsey 1991, Wolsey 2002) that a good understanding of the polyhedral structure of single-item lot-sizing problems can be very useful in solving more complicated problems, involving multiple products and stages. Because the single-item lot-sizing polyhedron is contained as a fundamental substructure in those problems, investigating inventory bounds and fixed costs within the lot-sizing context is meaningful from a practical point of view.

Although the polyhedron of lot sizing with inventory bounds and fixed costs is of interest in its own right, a secondary goal in this study is to gain a better understanding of the polyhedral structure of paths in capacitated fixedcharge networks. In this context, due to its simple path network representation, the lot-sizing problem with inventory bounds and fixed costs is the first natural problem to investigate.

\section{Relevant Literature}

The lot-sizing problem lies at the core of production/order and inventory-planning applications and has been studied extensively. Wagner and Whitin (1958) give an $O\left(n^{2}\right)$ algorithm for the lot-sizing problem with no bounds on order quantity or inventory (uncapacitated lot-sizing problem), where $n$ is the number of time periods. Federgruen and Tzur (1991), Wagelmans et al. (1992), and Aggarwal and Park (1993) give $O(n \log n)$ algorithms for this problem.

The first polyhedral study of the uncapacitated lot-sizing problem is due to Bárány et al. (1984). They give a complete linear description of the convex hull of the solutions. Variants of the uncapacitated problem that include sales and safety stocks (Loparic et al. 2001), order/production lower bounds (Constantino 1998), backlogging and startups (Pochet and Wolsey 1988, Constantino 1996, Agra and Constantino 1999), and piecewise linear concave costs (Aghezzaf and Wolsey 1994) have been studied. 
The lot-sizing problem with upper bounds on production/order quantities is $\mathcal{N} \mathscr{P}$-hard (Florian et al. 1971). Pochet (1988), Miller et al. (2000), Loparic et al. (2003), and Atamtürk and Muñoz (2004) give inequalities for lot sizing with order capacities and uncapacitated inventory. If order capacities are constant, the problem can be solved in polynomial time (Florian and Klein 1980, Van Hoesel and Wagelmans 1996). Leung et al. (1989) and Pochet and Wolsey (1993) describe inequalities for this case. However, a complete linear description of the convex hull of the solutions for the constant-capacity lot sizing is unknown. Atamtürk and Hochbaum (2001) study constant-capacity lot sizing with capacity acquisition and subcontracting.

Belvaux and Wolsey $(2000,2001)$ discuss strong formulations and a specialized branch-and-cut system for practical lot-sizing problems. Recently, van Vyve and Ortega (2003) gave a linear description of the convex hull of the solutions to uncapacitated lot sizing with inventory fixed costs. This is the only polyhedral study we are aware of that considers inventory fixed costs.

In all of the studies mentioned above, inventory is assumed to be uncapacitated. Love (1973) proposes a polynomial algorithm for the lot-sizing problem with unbounded order quantity and bounded inventory. To the best of our knowledge, the only polyhedral study on lot sizing that considers inventory capacities is Pochet and Wolsey (1994), in which the authors study lot-sizing problems under WagnerWhitin nonspeculative cost structure. They give a linear programming formulation of lot-sizing problems with uncapacitated and constant-capacity production and inventory upper bounds.

Lot-sizing problems are special fixed-charge network flow problems. For uncapacitated fixed-charge network flows, Van Roy and Wolsey (1985) give inequalities that are based on path substructures. Ortega and Wolsey (2000) present a computational study on the performance of path and dicut inequalities in solving the uncapacitated fixed-charge network flow problem. Padberg et al. (1985), Stallaert (1997), Gu et al. (1999), and Atamtürk (2001) describe valid inequalities for capacitated fixed-charge networks based on single-node relaxations. These inequalities do not capture the path substructure of capacitated networks. Submodular inequalities of Wolsey (1989) take into account capacities, but not the fixed charges on arcs along a path.

\section{Outline}

In $\S 2$, we formally present the lot-sizing problem with inventory bounds and fixed costs and introduce the notation used in the paper. Section 3 is devoted to the special case with linear inventory costs. In $\$ 4$, we study the polyhedron for linear and fixed inventory costs and extend the inequalities defined in $\$ 3$ to this more general case. We give polynomial exact separation algorithms for these inequalities. In addition, we give a linear programming formulation for the lot-sizing problem with inventory bounds and fixed costs if order and inventory costs satisfy the Wagner-Whitin nonspeculative property. Section 5 summarizes our computational experiments on testing the effectiveness of the inequalities when used as cuts for solving the problem. We conclude in $\$ 6$.

\section{Lot-Sizing Problem with Bounded Inventory}

For a finite planning horizon $n$, given the demand $d_{t}$, variable order cost $p_{t}$, and fixed order cost $f_{t}$ for time periods $t \in\{1,2, \ldots, n\}$; and inventory capacity $u_{t}$, variable inventory holding cost $h_{t}$, and fixed inventory holding cost $g_{t}$ for $t \in\{0,1, \ldots, n\}$; the lot-sizing problem with bounded inventory (LSBI) is to determine the order quantity and inventory in each period so that the sum of order and inventory holding costs over the horizon is minimized. We assume that order quantity is unbounded, although given the demand, there is an implicit upper bound on the order quantity due to the inventory capacities. We let $[i, j]:=$ $\{t \in \mathbb{Z}: i \leqslant t \leqslant j\}$ throughout.

Let $y_{t}$ denote the order quantity in time period $t$ and $i_{t}$ denote the inventory at the end of period $t$. Also, let $x_{t}$ and $z_{t}$ be the fixed-charge variables for order and inventory in period $t$, respectively. Then, LSBI can be formulated as

$$
\begin{aligned}
(\mathrm{LSBI}) \min & \sum_{t=1}^{n}\left(f_{t} x_{t}+p_{t} y_{t}+g_{t} z_{t}+h_{t} i_{t}\right)+g_{0} z_{0}+h_{0} i_{0} \\
\text { s.t. } i_{t-1}+y_{t}-i_{t}=d_{t}, \quad t \in[1, n] & \\
& 0 \leqslant i_{t} \leqslant u_{t} z_{t}, \quad t \in[0, n], \\
& 0 \leqslant y_{t} \leqslant\left(d_{t}+u_{t}\right) x_{t}, \quad t \in[1, n], \\
y & \in \mathbb{R}^{n}, \quad x \in\{0,1\}^{n}, \quad i \in \mathbb{R}^{n+1}, \\
& z \in\{0,1\}^{n+1} .
\end{aligned}
$$

Let $d_{t l}=\sum_{j=t}^{l} d_{j}$ for $t \in[1, l]$ and $d_{t l}=0$ for $t>l$. We let $\mathbb{Q}$ denote the convex hull of the feasible solutions to LSBI. Observe that for the optimization problem LSBI, if desired, one may ensure that $i_{0}=i_{n}=0$ in an optimal solution by assigning sufficiently high values to $h_{0}$ and $h_{n}$. Therefore, for generality, we keep $i_{0}$ and $i_{n}$ in the formulation for the polyhedral analysis. Throughout the paper, we assume that the data of the model consists of rational numbers and satisfy the following:

Assumption 1. $u_{t}>0$ for $t \in[0, n]$.

Assumption 2. $u_{t-1} \geqslant d_{t}$ for $t \in[1, n]$.

ASSUMPTION 3. $u_{t-1} \leqslant d_{t}+u_{t}$ for $t \in[1, n]$.

AssumPTION 4. $d_{t} \geqslant 0$ for $t \in[1, n]$.

Assumptions 1 and 3 are made without loss of generality. None of the validity proofs use Assumption 2. Therefore, all inequalities in this paper are valid without Assumption 2, which is used only in facet proofs for convenience. If $u_{t}=0$ for $t \in[0, n]$, then $i_{t}=0$ and the problem decomposes into two subproblems for $t \in[1, n-1]$. 
If $u_{t-1}<d_{t}$ for $t \in[1, n]$, then $x_{t}=1$ in every feasible solution. If $u_{t-1}>d_{t}+u_{t}$ for $t \in[1, n]$, then $u_{t-1}$ can be reduced to $d_{t}+u_{t}$ without changing the feasible set of solutions. Finally, $d_{t}<0$ does not make sense for the lot-sizing problem.

Definition 1. For a given point $(y, x, i, z)$ in $\mathbb{Q}$, a consecutive sequence of time periods $[k, l]$ is called a block if $i_{k-1} \in\left\{0, u_{k-1}\right\}, i_{l} \in\left\{0, u_{l}\right\}$, and $0<i_{t}<u_{t}$ for all $t \in[k, l-1]$.

The block definition leads to a characterization of the extreme points of $\mathbb{Q}$ and a polynomial dynamic programming algorithm (Love 1973) for LSBI, which can be implemented in $O\left(n^{2}\right)$. We represent the four types of blocks by $[k, l]_{\beta}^{\alpha}$, where $\alpha \in\left\{0, u_{k-1}\right\}$ and $\beta \in\left\{0, u_{l}\right\}$. It follows from the network structure of the problem that $(y, x, i, z)$ is an extreme point of $\mathbb{Q}$ if and only if there is at most one period with positive order quantity in every block $[k, l]$. Consequently, the order quantity in such a period equals $d_{k l}+\beta-\alpha$.

Example 1. Suppose that LSBI is given as $\left(d_{1}, \ldots, d_{5}\right)=$ $(11,12,13,14,15)$ and $\left(u_{0}, \ldots, u_{5}\right)=(\infty, 30, \infty, \infty$, $\infty, 5)$. Then, we can strengthen the inventory bounds as $\left(u_{0}, \ldots, u_{5}\right)=(41,30,47,34,20,5)$ to satisfy Assumption 3. Figure 1 illustrates the four types of blocks in different extreme point solutions.

The linear programming (LP) relaxation of LSBI has the same block structure; however, the fixed-charge variables take the values $x_{t} \in\left\{1, y_{t} /\left(d_{t}+u_{t}\right)\right\}$ and $z_{t} \in\left\{1, i_{t} / u_{t}\right\}$ and are typically highly fractional. The LP extreme point solution in Figure $1(\mathrm{a})$ has $\left(x_{1}, \ldots, x_{5}\right)=(1,0,0,24 / 34,0)$ and $\left(z_{1}, \ldots, z_{5}\right)=(0,1,18 / 47,5 / 34,3 / 4,0)$.
In $\$ 3$, we consider the case with linear costs on inventory, i.e., the restriction of $\mathbb{Q}$, where inventory fixed-charge variables are one. We describe valid inequalities that cut off all extreme points of the LP relaxation with fractional order fixed-charge variables. In $\S 4$, we generalize these inequalities to incorporate the inventory fixed-charge variables as well.

Notation. We introduce the following notation, which will be used throughout the paper: For $1 \leqslant k \leqslant l \leqslant n$, let:

$$
\begin{aligned}
& p=\min \left\{t \in[k, l]: d_{k t}>u_{k-1}\right\} \quad\left(p=l+1 \text { if } u_{k-1} \geqslant d_{k l}\right) \\
& q=\min \left\{t \in[k, l]: d_{k t} \geqslant u_{k-1}\right\} \quad\left(q=l+1 \text { if } u_{k-1}>d_{k l}\right), \\
& r=\max \left\{t \in[k, p]: u_{t}<d_{(t+1) l}\right\} \quad\left(r=k-1 \text { if } u_{t} \geqslant\right. \\
& \left.d_{(t+1) l} \text { for all } t \in[k, p]\right) \text {. }
\end{aligned}
$$

\section{Linear Inventory Costs}

In this section, we address the special case of LSBI with linear holding costs. When $g=0$, the convex hull of the feasible set of interest is $\mathscr{P}=\{(y, x, i, z) \in \mathbb{Q}: z=1\}$ or

$$
\mathscr{P}=\operatorname{conv}\left\{\begin{array}{ll}
i_{t-1}+y_{t}-i_{t}=d_{t}, & t \in[1, n], \\
0 \leqslant i_{t} \leqslant u_{t}, & t \in[0, n], \\
0 \leqslant y_{t} \leqslant\left(d_{t}+u_{t}\right) x_{t}, & t \in[1, n], \\
y \in \mathbb{R}^{n}, x \in\{0,1\}^{n}, & i \in \mathbb{R}^{n+1} .
\end{array}\right\}
$$

\subsection{Uncapacitated Inequalities}

For the special case of $\mathscr{P}$ with no inventory capacities, Bárány et al. (1984) give the so-called $(l, S)$ inequalities

$\sum_{t \in S} y_{t} \leqslant \sum_{t \in S} d_{t l} x_{t}+i_{l}, \quad$ where $S \subseteq[1, l]$ and $l \in[1, n]$.

They show that adding inequalities (1) to the LP relaxation suffices to describe the convex hull of feasible solutions. Example 1 illustrates that in the presence of inventory

Figure 1. Four types of blocks in extreme point solutions of $\mathbb{Q}$.

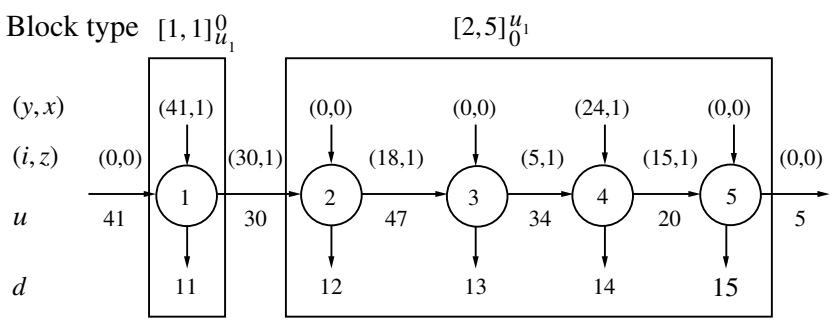

(a)

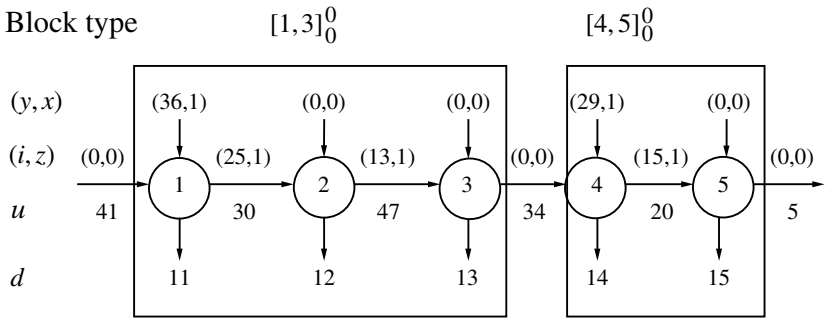

(c)

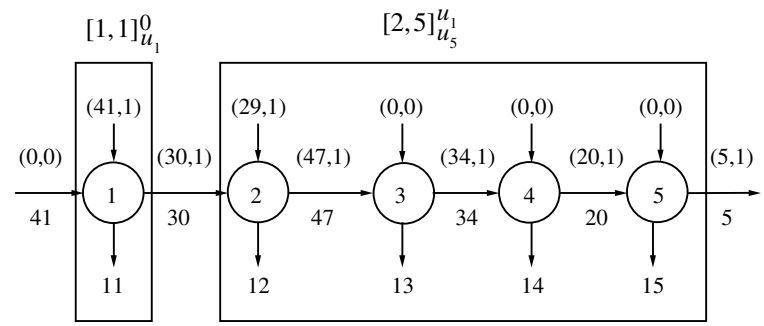

(b)

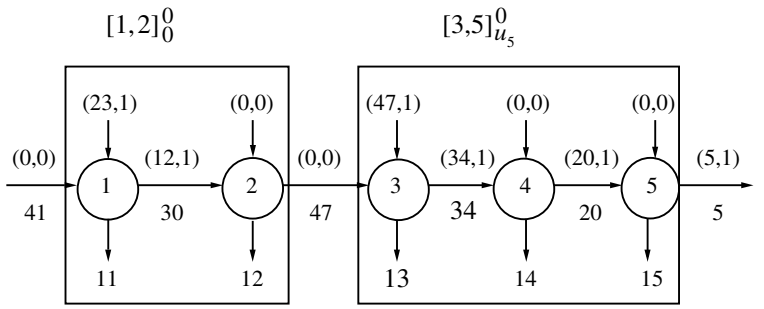

(d) 
upper bounds $(l, S)$ inequalities may be weak and are not sufficient to describe $\mathscr{P}$.

EXAMPLE 1 (continued). It is easy to check that the extreme point solution of the LP relaxation with $\left(x_{1}, \ldots, x_{5}\right)=$ $(1,29 / 59,0,0,0)$ (in Figure 1(b)) cannot be cut off by any $(l, S)$ inequality $(1)$. Observe that the $(l, S)$ inequality

$y_{1}+\cdots+y_{5} \leqslant 65 x_{1}+54 x_{2}+42 x_{3}+29 x_{4}+15 x_{5}+i_{5}$,

with $l=5$ and $S=[1,5]$, can be strengthened as

$y_{1}+\cdots+y_{5} \leqslant 41 x_{1}+54 x_{2}+42 x_{3}+29 x_{4}+15 x_{5}+i_{5}$

because $y_{1} \leqslant 11+30$. However, this inequality is dominated by the variable upper-bound constraint $y_{1} \leqslant 41 x_{1}$ and inequality (1) with $l=5$ and $S=[2,5]$. Thus, if $d_{t}+u_{t}<d_{t l}$ for some $t \in S$, then inequality (1) and even the strengthening of (1),

$\sum_{t \in S} y_{t} \leqslant \sum_{t \in S} \min \left\{d_{t l}, d_{t}+u_{t}\right\} x_{t}+i_{l}$,

are weak.

\subsection{Capacitated Inequalities}

In $\S 3.1$, we have illustrated that $(l, S)$ inequality (1) may not cut off fractional LP extreme solutions if for a block incoming or outgoing inventory is at capacity. Motivated by this observation, we obtain new inequalities by saturating incoming and outgoing inventory variables for a block.

ExAmple 1 (continued). To derive a strong inequality that uses inventory upper bounds, we observe that due to $u_{1}$, it is not possible to meet the total demand $d_{2}+d_{3}+d_{4}=39$ from inventory $i_{1}$. Therefore, an order must be placed in Periods 2,3 , or 4 , which is stated by the inequality

$x_{2}+x_{3}+x_{4} \geqslant 1$,

cutting off the fractional solution in Figure 1(b) that cannot be cut by the uncapacitated inequalities.

In general, for any $1 \leqslant k \leqslant l \leqslant n$ such that $u_{k-1}<d_{k l}$, the "cut-set type" inequality

$\sum_{t \in[k, l]} x_{t} \geqslant 1$

is valid for $\mathscr{P}$. Note that if $u_{k-1}<d_{k(l-1)}$, then (2) is dominated by $\sum_{t \in[k, l-1]} x_{t} \geqslant 1$; therefore, strong inequalities among (2) must satisfy $d_{k(l-1)} \leqslant u_{k-1}<d_{k l}$, implying that there are only at most $n$ strong inequalities among (2). Next, we introduce inequalities that generalize (2).

The first class of inequalities is obtained by setting the incoming inventory variable $i_{k-1}$ for $[k, l]$ to $u_{k-1}$ for $u_{k-1} \leqslant d_{k l}$ (Figure 2). Then, due to the exogenous supply $u_{k-1}$ in time period $k$, the effective total demand in periods $t, t+1, \ldots, l$ is $\min \left\{d_{k l}-u_{k-1}, d_{t l}\right\}$ for $t \in[k, l]$. Also
Figure 2. Saturating incoming inventory.

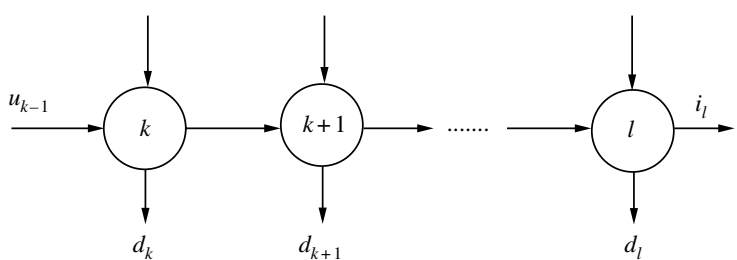

observing, in this case, that the order quantity in period $t$ cannot exceed $d_{k t}-u_{k-1}+u_{t}$ due to the inventory capacity $u_{t}$, we obtain the following inequalities.

For $1 \leqslant k \leqslant l \leqslant n$ such that $u_{k-1} \leqslant d_{k l}$ and $S \subseteq[k, l]$, consider the inequality

$$
\begin{aligned}
& i_{k-1}+\sum_{t \in S} y_{t} \\
& \leqslant u_{k-1}+\sum_{t \in S} \min \left\{d_{k t}+u_{t}-u_{k-1}, d_{k l}-u_{k-1}, d_{t l}\right\} x_{t}+i_{l} .
\end{aligned}
$$

Proposition 1. Inequality (3) is valid for $\mathscr{P}$.

PROOF. If $u_{k-1}=d_{k l}$, inequality follows from $i_{k-1}+$ $\sum_{t \in S} y_{t} \leqslant d_{k l}+i_{l}=u_{k-1}+i_{l}$. Otherwise, let $p=\min \{t \in$ $\left.[k, l]: u_{k-1}<d_{k t}\right\}$. Observe that $d_{k l}-u_{k-1} \leqslant d_{t l}$ for $t \in[k, p]$ and $d_{k l}-u_{k-1}>d_{t l}$ for $t \in[p+1, l]$. For $(y, x, i) \in \mathscr{P}$, let $b=\max \left\{t \in S: d_{k t}+u_{t}-u_{k-1}<\min \left\{d_{k l}-\right.\right.$ $\left.u_{k-1}, d_{t l}\right\}$ and $\left.x_{t}=1\right\}$ (if no such $t$ exists, let $b=k-1$ ) and let $h=\min \left\{t \in[b+1, l] \cap S: x_{t}=1\right\}$ (if no such $t$ exists, let $h=l+1$ ). If $h \leqslant p$, then

$$
\begin{aligned}
& i_{k-1}+\sum_{t \in S} y_{t} \\
& \quad \leqslant d_{k l}+i_{l}=d_{k b}+d_{(b+1) l}+u_{b}-u_{b}+u_{k-1}-u_{k-1}+i_{l} \\
& \quad \leqslant u_{k-1}+\sum_{t \in S} \min \left\{d_{k t}+u_{t}-u_{k-1}, d_{k l}-u_{k-1}, d_{t l}\right\} x_{t}+i_{l} .
\end{aligned}
$$

The last inequality follows because $x_{h}=1, d_{k l}-u_{k-1} \leqslant d_{h l}$ for $h \leqslant p, d_{(b+1) l}-u_{b} \leqslant d_{k l}-u_{k-1}$ (by Assumption 3), $d_{k l}-u_{k-1} \geqslant 0$, and $d_{k t}+u_{t}-u_{k-1} \geqslant 0$ (by Assumption 3).

On the other hand, if $h>p$, then

$$
\begin{aligned}
& i_{k-1}+\sum_{t \in S} y_{t} \\
& \leqslant d_{k b}+u_{b}+d_{h l}+i_{l}+u_{k-1}-u_{k-1} \\
& \leqslant u_{k-1}+\sum_{t \in S} \min \left\{d_{k t}+u_{t}-u_{k-1}, d_{k l}-u_{k-1}, d_{t l}\right\} x_{t}+i_{l} .
\end{aligned}
$$

REMARK 1. Note that Assumption 2 is not used to prove the validity of inequality (3). If $u_{k-1}<d_{k}$ for some $k \in$ $[1, n]$, then we write inequality (3) for $k=l$ and $S=\{k\}$ as

$i_{k-1}+y_{k} \leqslant u_{k-1}+\left(d_{k}-u_{k-1}\right) x_{k}+i_{k}$.

Adding the flow balance equality $d_{k}+i_{k}=y_{k}+i_{k-1}$, we get $d_{k}-u_{k-1} \leqslant\left(d_{k}-u_{k-1}\right) x_{k}$ or $x_{k} \geqslant 1$. 
Remark 2. If there exists $j \in S \cap[p+1, l]$ with $u_{j}<$ $d_{(j+1) l}$, then inequality (3) can be strengthened as

$$
\begin{gathered}
i_{k-1}+\sum_{t \in S} y_{t} \leqslant u_{k-1}+\sum_{t \in S} \min \left\{d_{k t}+u_{t}-u_{k-1}, d_{k l}-u_{k-1},\right. \\
\left.d_{t l}, d_{t}+u_{t}\right\} x_{t}+i_{l} .
\end{gathered}
$$

However, inequality (4) is weak, because it is dominated by

$$
\begin{aligned}
& i_{k-1}+\sum_{t \in S \backslash\{j\}} y_{t} \\
& \leqslant u_{k-1}+\sum_{t \in S \backslash\{j\}} \min \left\{d_{k t}+u_{t}-u_{k-1}, d_{k l}-u_{k-1}, d_{t l}\right\} x_{t}+i_{l},
\end{aligned}
$$

and the constraint $y_{j} \leqslant\left(d_{j}+u_{j}\right) x_{j}$. It follows that $u_{j} \geqslant$ $d_{(j+1) l}$ for $j \in[p+1, l]$ is a necessary facet condition for inequality (3); see Proposition 13.

Remark 3. We note that inequality (3) dominates inequality (2). To see this, suppose that $u_{k-1}<d_{k l}$, hence, inequality (2) is valid for $\mathscr{P}$. Rewriting inequality (3) for $S=[k, l]$ by subtracting the aggregate flow balance equality

$i_{k-1}+\sum_{t \in[k, l]} y_{t}=d_{k l}+i_{l}$

we obtain

$\sum_{t \in[k, l]} \min \left\{d_{k t}+u_{t}-u_{k-1}, d_{k l}-u_{k-1}, d_{t l}\right\} x_{t} \geqslant d_{k l}-u_{k-1}$,

which is at least as strong as inequality (2).

The next class of inequalities is obtained by saturating incoming as well as outgoing inventory variables $i_{k-1}$ and $i_{l}$ for a block $[k, l]$ (Figure 3). Then, due to the exogenous supply in time period $k$, the effective total demand in periods $t, t+1, \ldots, l$ is $\min \left\{d_{k l}-u_{k-1}+u_{l}, d_{t l}+u_{l}\right\}$ for $t \in[k, l]$. However, observing in this case that the order quantity in period $t$ cannot exceed $d_{k t}-u_{k-1}+u_{t}$ due to the inventory capacity $u_{t}$, and that $d_{k t}-u_{k-1}+u_{t} \leqslant$ $d_{k l}-u_{k-1}+u_{l}$ by Assumption 3, we obtain the following inequalities.

For $k \geqslant 1$ and $S \subseteq[k, n]$, consider the inequality

$$
i_{k-1}+\sum_{t \in S} y_{t} \leqslant u_{k-1}+\sum_{t \in S}\left(d_{k t}-u_{k-1}+u_{t}\right) x_{t} .
$$

Proposition 2. Inequality (5) is valid for $\mathscr{P}$.

Figure 3. Saturating incoming and outgoing inventory.

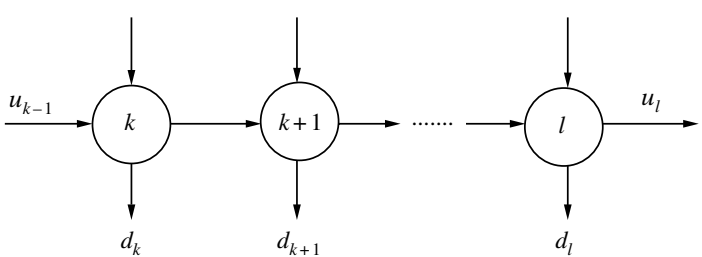

Proof. Let $(y, x, i) \in \mathscr{P}$. If $x_{t}=0$ for all $t \in S$, then inequality is trivially valid. Otherwise, let $\omega=\max \{t \in$ $\left.S: x_{t}=1\right\}$. Then,

$$
\begin{aligned}
i_{k-1}+\sum_{t \in S} y_{t} & \leqslant d_{k \omega}+u_{\omega}+u_{k-1}-u_{k-1} \\
& \leqslant u_{k-1}+\sum_{t \in S}\left(d_{k t}+u_{t}-u_{k-1}\right) x_{t} .
\end{aligned}
$$

Remark 4. Suppose that $S \subseteq[k, l]$ for $l \geqslant k$. Then, inequality

$i_{k-1}+\sum_{t \in S} y_{t} \leqslant u_{k-1}+\sum_{t \in S} \min \left\{d_{k t}+u_{t}-u_{k-1}, d_{t l}+u_{l}\right\} x_{t}$

is dominated by inequality (5) with $S^{\prime}=S \cap[k, q]$, where $q=\min \left\{t \in[k, l]: d_{k t} \geqslant u_{k-1}\right\}$ and inequality $y_{t} \leqslant\left(d_{t}+\right.$ $\left.u_{t}\right) x_{t}$ for $t \in S \backslash S^{\prime}$ because $d_{t}+u_{t} \leqslant d_{t l}+u_{l}$ by Assumption 3. It follows that $S \subseteq[k, q]$ is a necessary facet condition for Inequality (5); see Proposition 14.

In the appendix we study the strength of inequalities (1), (3), and (5) with respect to $\mathscr{P}$

EXAmPLe 1 (continued). For $[k, l]=[2,5]$, we have $p=$ $q=4$. Then, all facet-defining inequalities (3) of $\mathscr{P}$ for $[k, l]=[2,5]$ with $|S| \leqslant 2$ are

$$
\begin{aligned}
& i_{1}+y_{2} \leqslant 30+24 x_{2}+i_{5}, \\
& i_{1}+y_{3} \leqslant 30+24 x_{3}+i_{5}, \\
& i_{1}+y_{4} \leqslant 30+24 x_{4}+i_{5}, \\
& i_{1}+y_{2}+y_{3} \leqslant 30+24 x_{2}+24 x_{3}+i_{5}, \\
& i_{1}+y_{2}+y_{4} \leqslant 30+24 x_{2}+24 x_{4}+i_{5}, \\
& i_{1}+y_{3}+y_{4} \leqslant 30+24 x_{3}+24 x_{4}+i_{5}, \\
& i_{1}+y_{2}+y_{5} \leqslant 30+24 x_{2}+15 x_{5}+i_{5}, \\
& i_{1}+y_{3}+y_{5} \leqslant 30+24 x_{3}+15 x_{5}+i_{5}, \\
& i_{1}+y_{4}+y_{5} \leqslant 30+24 x_{4}+15 x_{5}+i_{5} .
\end{aligned}
$$

On the other hand, all facet-defining inequalities (5) of $\mathscr{P}$ for $[k, l]=[2,5]$ are

$$
\begin{aligned}
& i_{1} \leqslant 30, \\
& i_{1}+y_{2} \leqslant 30+29 x_{2}, \\
& i_{1}+y_{3} \leqslant 30+29 x_{3}, \\
& i_{1}+y_{4} \leqslant 30+29 x_{4}, \\
& i_{1}+y_{2}+y_{3} \leqslant 30+29 x_{2}+29 x_{3}, \\
& i_{1}+y_{2}+y_{4} \leqslant 30+29 x_{2}+29 x_{4}, \\
& i_{1}+y_{3}+y_{4} \leqslant 30+29 x_{3}+29 x_{4} .
\end{aligned}
$$




\subsection{Fractional Extreme Points of the LP Relaxation}

Now we show that the inequalities described in $\S \S 3.1$ and 3.2 cut all fractional extreme point solutions of the linear programming relaxation of LSBI. Recall that extreme point solutions are characterized by blocks $[k, l]_{\beta}^{\alpha}$ for $1 \leqslant$ $k \leqslant l \leqslant n$, where $\alpha \in\left\{0, u_{k-1}\right\}$ and $\beta \in\left\{0, u_{l}\right\}$. Note that in an extreme point solution there can be at most one period with positive order quantity in a block.

Proposition 3. Inequalities (1), (3), and (5) cut all fractional extreme point solutions of the $L P$ relaxation of $L S B I$.

Proof. Let $(y, x, i)$ be an extreme point solution of the LP relaxation of LSBI. For a block type $[k, l]_{0}^{0}$, there exists one period $t \in[k, l]$ with $y_{t}=d_{t l}=d_{k l}$. (Note that $t=k$ if $d_{k}>0$.) Therefore, the order fixed-charge variable $x_{t}=$ $d_{k l} /\left(d_{t}+u_{t}\right)$. If $0<x_{t}<1$, then inequality (1),

$y_{t} \leqslant d_{t l} x_{t}+i_{l}$,

cuts off this point.

For a block type $[k, l]_{0}^{u_{k-1}}$, there exists one period $t \in$ $[k, l]$ with $y_{t}=d_{k l}-u_{k-1}$. Therefore, the order fixed-charge variable $x_{t}=\left(d_{k l}-u_{k-1}\right) /\left(d_{t}+u_{t}\right)$. If $0<x_{t}<1$, then inequality (3),

$i_{k-1}+y_{t} \leqslant u_{k-1}+\left(d_{k l}-u_{k-1}\right) x_{t}+i_{l}$,

cuts off $(y, x, i)$.

For a block type $[k, l]_{u_{l}}^{u_{k-1}}$, there exists one period $t \in$ $[k, l]$ with $y_{t}=d_{k l}+u_{l}-u_{k-1}$. Therefore, the order fixed-charge variable $x_{t}=\left(d_{k l}+u_{l}-u_{k-1}\right) /\left(d_{t}+u_{t}\right)$. If $0<x_{t}<1$, then inequality (5),

$i_{k-1}+y_{t} \leqslant u_{k-1}+\left(d_{k l}+u_{l}-u_{k-1}\right) x_{t}$,

cuts off $(y, x, i)$.

Finally, for a block type $[k, l]_{u_{l}}^{0}$, there exists one period $t \in[k, l]$ with $y_{t}=d_{k l}+u_{l}$. In this case, we must have $d_{k l}+u_{l}=d_{t}+u_{t}$. Therefore, the order fixed-charge variable is integral.

\subsection{Separation}

Now we discuss how to find inequality (3) violated by a given point $(y, x, i) \in \mathbb{R}_{+}^{3 n+1}$. Let $\Delta=\sum_{t \in S}\left(y_{t}-\min \left\{d_{k t}+\right.\right.$ $\left.\left.u_{t}-u_{k-1}, d_{k l}-u_{k-1}, d_{t l}\right\} x_{t}\right)$. For fixed $k$ and $l$ such that $u_{k-1}<d_{k l}, \Delta$ is maximized by placing $t \in[k, l]$ in $S$ if and only if $y_{t}>\min \left\{d_{k t}+u_{t}-u_{k-1}, d_{k l}-u_{k-1}, d_{t l}\right\} x_{t}$. Because this can be done in linear time for each $k$ and $l$, the observation leads to an $O\left(n^{3}\right)$ separation algorithm for inequality (3). Next, we improve the computational complexity of separation.

THEOREM 4. There is an $O\left(n^{2} \log n\right)$ algorithm to solve the separation problem for inequalities (3). There are $O(n \log n)$ algorithms to solve the separation problems for inequalities (1) and (5).
Proof. For $k \in[1, n-1]$, let $p(k)=\min \left\{t \in[k, l]: u_{k-1}<\right.$ $\left.d_{k t}\right\}\left(p(k)=l+1\right.$ if $\left.u_{k-1} \geqslant d_{k l}\right)$. Also, let $r^{\prime}=\max \{t \in$ $\left.[1, l]: u_{t}<d_{(t+1) l}\right\}$. Therefore, in inequality (3), $x_{t}$ for $t \in S$ has coefficient $d_{k t}+u_{t}-u_{k-1}$ if $t \in\left[k, \min \left\{r^{\prime}, p(k)\right\}\right], d_{k l}-$ $u_{k-1}$ if $t \in\left[r^{\prime}+1, p(k)\right]$, and $d_{t l}$ if $t \in\left[\max \left\{r^{\prime}, p(k)\right\}+\right.$ $1, l]$. (Observe that to obtain a strong inequality, we must have $S \cap\left[p(k)+1, r^{\prime}\right]=\varnothing$ for $r^{\prime}>p(k)$.)

In the rest of the discussion, we fix $l$ and decrement $k$ from $l$ to 1 . A set $S$ that maximizes $\Delta$ for all $k \in[1, l]$ will be computed in $O(n \log n)$. The following observations are due to Assumption 3: (a) $p(k-1) \leqslant p(k)$, and (b) $d_{k l}-$ $u_{k-1} \leqslant d_{(k-1) l}-u_{k-2}$.

Let $S(k)$ be a subset of $[k, l]$ maximizing $\Delta$ for $k$ (and fixed $l$ ). Observation (b) implies that if $t \in\left[k, r^{\prime}\right]$ and $r^{\prime} \leqslant$ $p(k)$ and $t \notin S(k)$, then $t \notin S(j)$ for any $j \leqslant k$; also, if $t \in\left[r^{\prime}+1, p(k)\right]$ and $t \notin S(k)$, then $t \notin S(j)$ for any $j \leqslant k$ such that $t \leqslant p(j)$. However, $t$ may be in $S(j)$ if $p(j)<t$. It is clear that if $t \in\left[\max \left\{r^{\prime}, p(k)\right\}+1, l\right]$ and $t \notin S(k)$, then $t \notin S(j)$ for any $j \leqslant k$. Also note that $S(j) \cap\left[p(j)+1, r^{\prime}\right]=$ $\varnothing$ for $r^{\prime}>p(k)$.

Let $T$ be the list of $t \in\left[k, r^{\prime}\right]$ for $r^{\prime} \leqslant p(k)$ such that $\psi_{t}:=y_{t} / x_{t}-\left(d_{k t}+u_{t}-u_{k-1}\right)>0$, sorted by $\psi_{t} ; T^{\prime}$ be the list of $t \in\left[r^{\prime}+1, p(k)\right]$ such that $\varepsilon_{t}:=y_{t} / x_{t}-\left(d_{k l}-\right.$ $\left.u_{k-1}\right)>0$, sorted by $\varepsilon_{t}$; and, finally, $T^{\prime \prime}$ be the (unsorted) list of $t \in\left[\max \left\{r^{\prime}, p(k)\right\}+1, l\right]$ such that $y_{t}-d_{t l} x_{t}>0$. For $k^{\prime}=k-1$, if $k^{\prime} \leqslant r^{\prime}$, then we place $k^{\prime}$ into $T$ in $O(\log n)$ time by binary search on $\psi_{t}$ and delete permanently from $T$ all $t \leqslant r^{\prime}$ with $\psi_{t}^{\prime}=\psi_{t}-\left(d_{k-1}-u_{k-2}+u_{k-1}\right) \leqslant 0$. On the other hand, if $r^{\prime}<k^{\prime}$, then we place $k^{\prime}$ into $T^{\prime}$ in $O(\log n)$ time by binary search on $\varepsilon_{t}$. We delete from $T^{\prime}$ all $t>p\left(k^{\prime}\right)$ and all $t \in T^{\prime}$ such that $\varepsilon_{t}^{\prime}=\varepsilon_{t}-\left(d_{k-1}-u_{k-2}+u_{k-1}\right) \leqslant 0$ permanently. Finally, $t \in\left[p\left(k^{\prime}\right)+1, p(k)\right]$ is placed in $T^{\prime \prime}$ permanently if and only if $y_{t}-d_{t l} x_{t}>0$. Thus, $S\left(k^{\prime}\right)=$ $T \cup T^{\prime} \cup T^{\prime \prime}$

Keeping separate sums $y(T)=\sum_{t \in T} y_{t}, x(T)=\sum_{t \in T} x_{t}$ and $y\left(T^{\prime}\right)=\sum_{t \in T^{\prime}} y_{t}, x\left(T^{\prime}\right)=\sum_{t \in T^{\prime}} x_{t}$ allows us to compute $\Delta$ for $S(k)$ in constant average time. Because variables are inserted into and deleted from each list at most once, all updates in $\Delta$ for the changes in the lists can be done in $O(n)$. For the elements remaining in the lists, updates can be done in constant time per iteration. For instance, let $\bar{T} \subseteq T$ be the set of elements that remain from one iteration to the next. Because the coefficients of $x_{i}$ for all $i \in \bar{T}$ reduce by $d_{k-1}-u_{k-2}+u_{k-1}$, the required change for them in $\Delta$ is $-\left(d_{k-1}-u_{k-2}+u_{k-1}\right) x(\bar{T})$.

There are at most $n$ insertions to $T$ and $T^{\prime}$, all of which take total $O(n \log n)$. Also, there are at most $n$ deletions from $T^{\prime}$ and insertions to $T^{\prime \prime}$, all of which can be done in total $O(n)$. Because $\Delta$ can be updated in a total of $O(n)$, the complexity of the algorithm is $O(n \log n)$ for each $l$, giving an overall $O\left(n^{2} \log n\right)$ separation algorithm for inequality (3).

From the above algorithm, it also follows that the separation for the simpler inequality (5) can be done in $O(n \log n)$ by fixing $l=n$ only. Separation for inequality (1) can be done similarly in $O(n \log n)$ by keeping the elements of $T^{\prime \prime}$ sorted by $y_{t} / x_{t}-d_{t l}$ and incrementing $l$ from 1 to $n$. 


\section{Fixed Costs on Inventory}

In this section, we expand the study to incorporate the inventory fixed-charge variables. Thus, the polyhedron studied in this section is

$\mathbb{Q}=\operatorname{conv}\left\{\begin{array}{ll}i_{t-1}+y_{t}-i_{t}=d_{t}, & t \in[1, n], \\ 0 \leqslant i_{t} \leqslant u_{t} z_{t}, & t \in[0, n], \\ 0 \leqslant y_{t} \leqslant\left(d_{t}+u_{t}\right) x_{t}, & t \in[1, n], \\ y \in \mathbb{R}^{n}, x \in\{0,1\}^{n}, & i \in \mathbb{R}^{n+1}, z \in\{0,1\}^{n+1} .\end{array}\right\}$

\subsection{Uncapacitated Inequalities}

As before, we first describe inequalities that do not consider the inventory upper bounds. For $t \in[0, n-1]$, let $b_{t}$ be the first time period after $t$ with positive demand, i.e., $b_{t}=$ $\min \left\{k \in[t+1, n]: d_{k}>0\right\}$. Then, because $d_{b_{t}}$ is either satisfied from inventory at $t$ or from an order in a later period up to $b_{t}$, inequality

$z_{t}+x_{t+1}+\cdots+x_{b_{t}} \geqslant 1$

is valid for $Q$.

For $0 \leqslant k \leqslant l \leqslant n$ and $S \subseteq[k, l]$, inequality

$i_{k-1}+\sum_{t \in S} y_{t} \leqslant d_{k l} z_{k-1}+\sum_{t \in S} d_{t l} x_{t}+i_{l}$,

where $i_{-1}$ and $z_{-1}$ are taken as 0 , generalizes (7) as well as the $(l, S)$ inequalities (1). When there are no inventory upper bounds, van Vyve and Ortega (2003) show that it suffices to add inequalities (8) to the LP relaxation of the uncapacitated lot-sizing problem with inventory fixed costs to obtain the convex hull of the feasible solutions. Inequalities (8) are not sufficient to describe $\mathbb{Q}$; however, they define facets of $\mathbb{Q}$, as shown in the appendix.

\subsection{Capacitated Inequalities}

To obtain strong inequalities that use the inventory fixed costs as well as the upper bounds, we introduce the inventory fixed-charge variables into inequalities (3) and (5).

For $1 \leqslant k \leqslant l \leqslant n$ such that $u_{k-1} \leqslant d_{k l}$, let $S \subseteq[k, l]$ and $T:=\left\{t_{1}, t_{2}, \ldots, t_{\tau}\right\} \subseteq[k-1, p-1]$. For $j \in T$, let $s(j)=$ $\min \{t \in S \cup\{l+1\}: t>j\}$. Consider the inequality

$$
\begin{aligned}
& i_{k-1}+\sum_{t \in S} y_{t}+\sum_{t \in T} \gamma_{t}\left(1-z_{t}\right) \\
& \leqslant u_{k-1}+\sum_{t \in S} \min \left\{d_{k t}+u_{t}-u_{k-1}, d_{k l}-u_{k-1}, d_{t l}\right\} x_{t}+i_{l},
\end{aligned}
$$

where

$$
\gamma_{t_{j}}= \begin{cases}u_{k-1}-d_{k t_{\tau}} & \text { if } j=\tau \text { and } s\left(t_{j}\right)>p, \\ d_{\left(t_{j}+1\right)\left(t_{(j+1)}\right)} & \text { if } j<\tau \text { and } s\left(t_{j}\right)=s\left(t_{(j+1)}\right), \\ d_{\left(t_{j}+1\right)\left(s\left(t_{j}\right)-1\right)} & \text { if }\left(j<\tau \text { and } s\left(t_{j}\right)<s\left(t_{(j+1)}\right)\right) \\ & \text { or }\left(j=\tau \text { and } s\left(t_{j}\right) \leqslant p\right) .\end{cases}
$$

EXAmple 1 (continued). Consider inequality (3) for $[k, l]=$ $[2,5]$ (where $p=4)$ and $S=\{2\}$ :

$i_{1}+y_{2} \leqslant 30+24 x_{2}+i_{5}$,

which is facet defining for the restriction $z_{t}=1$ for $t \in$ $[0,5]$. Now if $z_{2}=0$, then we observe that $i_{1}+y_{2} \leqslant$ $12=d_{2}$. Therefore,

$i_{1}+y_{2}+18\left(1-z_{2}\right) \leqslant 30+24 x_{2}+i_{5}$

is valid for $Q$, where $T=\{2\}, t_{\tau}=2$, and $s(2)=l+1=$ $6>p$, so $\gamma_{2}=u_{1}-d_{2}=18$.

Alternatively, for inequality (11), if $z_{3}=0$, then $i_{1}+y_{2} \leqslant$ $25=d_{23}$. Therefore,

$i_{1}+y_{2}+5\left(1-z_{3}\right) \leqslant 30+24 x_{2}+i_{5}$,

where $\gamma_{3}=u_{1}-d_{23}=5$. Now for (12), if $z_{2}=0$, then $i_{1}+y_{2}+5\left(1-z_{3}\right) \leqslant 12+5=d_{2}+\gamma_{3}$ and inequality

$i_{1}+y_{2}+13\left(1-z_{2}\right)+5\left(1-z_{3}\right) \leqslant 12+5=d_{2}+\gamma_{3}$,

which is equivalent to inequality (9) with $T=\{2,3\}$, $s(2)=s(3)=6$, and $\gamma_{2}=d_{3}=13$.

This example illustrates that $\gamma$ in (10) are sequencedependent lifting coefficients for $z_{t}, t \in T$. Next, we give a direct proof of validity.

Proposition 5. Inequality (9) is valid for Q.

Proof. Let $(y, x, i, z) \in \mathbb{Q}$. If $z_{t}=1$ for all $t \in T$, validity follows from Proposition 1 . Therefore, we assume that $z_{t}=0$ for some $t \in T$. For a given point $(y, x, i, z) \in \mathbb{Q}$, for $1 \leqslant k \leqslant l \leqslant n, S \subseteq[k, l]$ and $T \subseteq[k-1, p-1]$, if $z_{t}=0$ for some $t \in T$, then let $V=\left\{v_{1}, v_{2}, \ldots, v_{\kappa}\right\}=$ $\cup_{j \in S \cup\{l+1\}}\left(\min \left\{t \in T: z_{t}=0, s(t)=j\right\}\right)$. Without loss of generality, assume that $v_{1}<v_{2}<\cdots<v_{\kappa} ; v_{0}=0$ and $v_{\kappa+1}=n$. Thus, $S \cap[s, p-1]$ is partitioned as $\left(S_{1}, S_{2}, \ldots, S_{\kappa}, S_{\kappa+1}\right)$, where $S_{j}=\left\{t \in S: v_{j-1}<t \leqslant v_{j}\right\}$, and $T$ is partitioned as $\left(T_{1}, T_{2}, \ldots, T_{\kappa}, T_{\kappa+1}\right)$, where $T_{j}=$ $\left\{t \in T: s\left(v_{j-1}\right) \leqslant t<s\left(v_{j}\right)\right\}$ with $s\left(v_{0}\right)=k-1$ and $s\left(v_{\kappa+1}\right)=n$. Observe that for $t_{j} \in T$ if $s\left(t_{j}\right) \leqslant p$,

$\sum_{t \in T \cap\left[t_{j}, s\left(t_{j}\right)-1\right]} \gamma_{t} \leqslant d_{\left(t_{j}+1\right)\left(s\left(t_{j}\right)-1\right)}$

and if $s\left(t_{j}\right)>p$,

$\sum_{t \in T \cap\left[t_{j}, s\left(t_{j}\right)-1\right]} \gamma_{t} \leqslant u_{k-1}-d_{k t_{j}}$

First, we express the left-hand side of (9) as the sum of $\kappa+1$ terms: $i_{k-1}+\sum_{t \in S: t \leqslant v_{1}} y_{t}+\sum_{t \in T: t<s\left(v_{1}\right)} \gamma_{t}\left(1-z_{t}\right)$, $\sum_{t \in S: v_{j-1}<t \leqslant v_{j}} y_{t}+\sum_{t \in T: s\left(v_{j-1}\right) \leqslant t<s\left(v_{j}\right)} \gamma_{t}\left(1-z_{t}\right)$ for $j \in[2, \kappa]$ and $\sum_{t \in S: t>v_{\kappa}} y_{t}$. Next, we show the following relations for the first $\kappa$ of these terms. 
Observe from (13) that if $s\left(v_{1}\right) \leqslant p$,

$$
\begin{aligned}
& i_{k-1}+\sum_{t \in S: t \leqslant v_{1}} y_{t}+\sum_{t \in T: t<s\left(v_{1}\right)} \gamma_{t}\left(1-z_{t}\right) \\
& \quad \leqslant d_{k v_{1}}+d_{\left(v_{1}+1\right)\left(s\left(v_{1}\right)-1\right)}=d_{k\left(s\left(v_{1}\right)-1\right)},
\end{aligned}
$$

because $i_{k-1}+\sum_{t \in S: t \leqslant v_{1}} y_{t} \leqslant d_{k v_{1}}$ when $z_{v_{1}}=i_{v_{1}}=0$. Similarly, if $s\left(v_{1}\right)>p$ (in which case $\left.\kappa=1\right)$, then

$$
\begin{aligned}
& i_{k-1}+\sum_{t \in S: t \leqslant v_{1}} y_{t}+\sum_{t \in T: t<s\left(v_{1}\right)} \gamma_{t}\left(1-z_{t}\right) \\
& \quad \leqslant d_{k v_{1}}+d_{v_{1} t_{\tau}}+u_{k-1}-d_{k t_{\tau}}=u_{k-1} .
\end{aligned}
$$

For $v_{j}, j \in[2, \kappa]$ with $s\left(v_{j}\right) \leqslant p$, we have

$$
\begin{aligned}
& \sum_{t \in S: v_{j-1}<t \leqslant v_{j}} y_{t}+\sum_{t \in T: s\left(v_{j-1}\right) \leqslant t<s\left(v_{j}\right)} \gamma_{t}\left(1-z_{t}\right) \\
& \quad \leqslant d_{\left(s\left(v_{j-1}\right)\right) v_{j}}+d_{\left(v_{j}+1\right)\left(s\left(v_{j}\right)-1\right)} \\
& \quad=d_{\left(s\left(v_{j-1}\right)\right)\left(s\left(v_{j}\right)-1\right)} .
\end{aligned}
$$

On the other hand, for $v_{\kappa}$ if $\kappa \geqslant 2$ and $s\left(v_{\kappa}\right)>p$, then

$$
\begin{aligned}
& \sum_{t \in S: v_{\kappa-1} \leqslant t \leqslant v_{\kappa}} y_{t}+\sum_{t \in T: s\left(v_{\kappa-1}\right) \leqslant t<s\left(v_{\kappa}\right)} \gamma_{t}\left(1-z_{t}\right) \\
& \quad \leqslant d_{s\left(v_{\kappa-1}\right) v_{\kappa}}+d_{\left(v_{\kappa}+1\right) t_{\tau}}+u_{k-1}-d_{k t_{\tau}} \\
& \quad=u_{k-1}-d_{k\left(s\left(v_{\kappa-1}\right)-1\right)} .
\end{aligned}
$$

To see the validity of inequality (9), we consider six cases. Let $b$ and $h$ be defined as in the proof of Proposition 1. First, suppose that $h \leqslant p$. If $s\left(v_{\kappa}\right)>p$, then from (15)-(18), we have

$$
\begin{aligned}
& i_{k-1}+\sum_{t \in S: t \leqslant v_{\kappa}} y_{t}+\sum_{t \in T} \gamma_{t}\left(1-z_{t}\right)+\sum_{t \in S: t>v_{\kappa}} y_{t} \\
& \quad \leqslant u_{k-1}+\sum_{t \in S: t>v_{\kappa}} y_{t} \\
& \quad \leqslant u_{k-1}+d_{(p+1) l}+i_{l} \\
& \left.\quad \leqslant u_{k-1}+d_{k l}-u_{k-1}+i_{l} \quad \text { (because } u_{k-1}<d_{k p}\right) \\
& \quad \leqslant u_{k-1}+\sum_{t \in S} \min \left\{d_{k t}+u_{t}-u_{k-1}, d_{k l}-u_{k-1}, d_{t l}\right\} x_{t}+i_{l},
\end{aligned}
$$

where the second inequality follows, because $\sum_{t \in S: t>v_{\kappa}} y_{t} \leqslant$ $d_{(p+1) l}+i_{l}$ if $s\left(v_{\kappa}\right)>p$ and the last inequality follows, because $x_{t} \geqslant 0$ for $t \in S, x_{h}=1$, and for $h \leqslant p, d_{k l}-u_{k-1} \leqslant$ $\min \left\{d_{k t}+u_{t}-u_{k-1}, d_{t l}\right\}$.

Similarly, if $s\left(v_{\kappa}\right) \leqslant p$, then from (15) and (17),

$$
\begin{aligned}
i_{k-1} & +\sum_{t \in S: t \leqslant v_{\kappa}} y_{t}+\sum_{t \in T} \gamma_{t}\left(1-z_{t}\right)+\sum_{t \in S: t>v_{\kappa}} y_{t} \\
& \leqslant d_{k\left(s\left(v_{k}\right)-1\right)}+d_{s\left(v_{k}\right) l}+i_{l} \\
& =d_{k l}+u_{k-1}-u_{k-1}+i_{l} \\
& \leqslant u_{k-1}+\sum_{t \in S} \min \left\{d_{k t}+u_{t}-u_{k-1}, d_{k l}-u_{k-1}, d_{t l}\right\} x_{t}+i_{l} .
\end{aligned}
$$

Now suppose that $h>p$. If $s\left(v_{\kappa}\right)>p$ and $b>v_{\kappa}$, then

$$
\begin{aligned}
i_{k-1} & +\sum_{t \in S: t \leqslant v_{\kappa}} y_{t}+\sum_{t \in T} \gamma_{t}\left(1-z_{t}\right)+\sum_{t \in S: v_{\kappa}<t \leqslant b} y_{t}+\sum_{t \in S: t \geqslant h} y_{t} \\
& \leqslant u_{k-1}+d_{s\left(v_{\kappa}\right) b}+u_{b}+d_{h l}+i_{l} \\
& =u_{k-1}+d_{k b}+u_{b}-u_{k-1}+d_{h l}+i_{l}+\left(u_{k-1}-d_{k\left(s\left(v_{\kappa}\right)-1\right)}\right) \\
& \leqslant u_{k-1}+\sum_{t \in S} \min \left\{d_{k t}+u_{t}-u_{k-1}, d_{k l}-u_{k-1}, d_{t l}\right\} x_{t}+i_{l},
\end{aligned}
$$

where the last inequality follows because $u_{k-1}<d_{k\left(s\left(v_{k}\right)-1\right)}$ for $s\left(v_{\kappa}\right)>p$. If $s\left(v_{\kappa}\right) \leqslant p$ and $b>v_{\kappa}$, then

$$
\begin{aligned}
& i_{k-1}+\sum_{t \in S: t \leqslant v_{\kappa}} y_{t}+\sum_{t \in T} \gamma_{t}\left(1-z_{t}\right)+\sum_{t \in S: v_{k}<t \leqslant b} y_{t}+\sum_{t \in S: t \geqslant h} y_{t} \\
& \quad \leqslant d_{k\left(s\left(v_{\kappa}\right)-1\right)}+d_{s\left(v_{k}\right) b}+u_{b}+d_{h l}+i_{l}+u_{k-1}-u_{k-1} \\
& \quad \leqslant u_{k-1}+\sum_{t \in S} \min \left\{d_{k t}+u_{t}-u_{k-1}, d_{k l}-u_{k-1}, d_{t l}\right\} x_{t}+i_{l} .
\end{aligned}
$$

If $s\left(v_{\kappa}\right)>p$ and $b \leqslant v_{\kappa}$, then

$$
\begin{aligned}
& i_{k-1}+\sum_{t \in S: t \leqslant v_{\kappa}} y_{t}+\sum_{t \in T} \gamma_{t}\left(1-z_{t}\right)+\sum_{t \in S: t \geqslant h} y_{t} \\
& \quad \leqslant u_{k-1}+d_{h l}+i_{l} \\
& \quad \leqslant u_{k-1}+\sum_{t \in S} \min \left\{d_{k t}+u_{t}-u_{k-1}, d_{k l}-u_{k-1}, d_{t l}\right\} x_{t}+i_{l} .
\end{aligned}
$$

Finally, if $s\left(v_{\kappa}\right) \leqslant p$ and $b \leqslant v_{\kappa}$, then

$$
\begin{aligned}
& i_{k-1}+\sum_{t \in S: t \leqslant v_{\kappa}} y_{t}+\sum_{t \in T} \gamma_{t}\left(1-z_{t}\right)+\sum_{t \in S: t>v_{\kappa}} y_{t} \\
& \leqslant d_{k\left(s\left(v_{\kappa}\right)-1\right)}+d_{h l}+i_{l} \\
& \leqslant u_{k-1}+\sum_{t \in S} \min \left\{d_{k t}+u_{t}-u_{k-1}, d_{k l}-u_{k-1}, d_{t l}\right\} x_{t}+i_{l} .
\end{aligned}
$$

In the next class of inequalities, we introduce inventory fixed-charge variables into inequality (5). For $1 \leqslant k \leqslant$ $l \leqslant n$, let $S \subseteq[k, l]$ and $T:=\left\{t_{1}, t_{2}, \ldots, t_{\tau}\right\} \subseteq[k-1, p-1]$, where $p=\min \left\{t \in[k, l]: u_{k-1}<d_{k t}\right\}$. For $t \in T$, let $s(j)=$ $\min \{t \in S \cup\{l+1\}: t>j\}$. Then, consider the inequality

$$
i_{k-1}+\sum_{t \in S} y_{t}+\sum_{t \in T} \gamma_{t}\left(1-z_{t}\right) \leqslant u_{k-1}+\sum_{t \in S}\left(d_{k t}+u_{t}-u_{k-1}\right) x_{t} \text {, }
$$

where $\gamma_{t}$ is defined as in (10).

Proposition 6. Inequality (19) is valid for Q.

Proof. For $(y, x, i, z) \in Q$ if $x_{t}=0$ for all $t \in S$, inequality follows from $i_{k-1}+\sum_{t \in T} \gamma_{t}\left(1-z_{t}\right) \leqslant u_{k-1}$ due to (15)-(18). Otherwise, let $\omega=\max \left\{t \in S: x_{t}=1\right\}$. Using the definitions in the proofs of Propositions 1 and 5 , if $s\left(v_{\kappa}\right)>p$ and $\omega>v_{\kappa}$, then

$$
\begin{gathered}
i_{k-1}+\sum_{t \in S: t \leqslant v_{\kappa}} y_{t}+\sum_{t \in T} \gamma_{t}\left(1-z_{t}\right)+\sum_{t \in S: v_{k}<t \leqslant \omega} y_{t} \\
\leqslant u_{k-1}+d_{s\left(v_{k}\right) \omega}+u_{\omega}+\left(d_{k p}-u_{k-1}\right) \\
\leqslant u_{k-1}+\sum_{t \in S}\left(d_{k t}+u_{t}-u_{k-1}\right) x_{t} .
\end{gathered}
$$


If $s\left(v_{\kappa}\right) \leqslant p$ and $\omega>v_{\kappa}$, then

$$
\begin{aligned}
i_{k-1}+ & \sum_{t \in S: t \leqslant v_{\kappa}} y_{t}+\sum_{t \in T} \gamma_{t}\left(1-z_{t}\right)+\sum_{t \in S: v_{\kappa}<t \leqslant \omega} y_{t} \\
& \leqslant d_{k\left(s\left(v_{\kappa}\right)-1\right)}+d_{s\left(v_{\kappa}\right) \omega}+u_{\omega}+u_{k-1}-u_{k-1} \\
& \leqslant u_{k-1}+\sum_{t \in S}\left(d_{k t}+u_{t}-u_{k-1}\right) x_{t} .
\end{aligned}
$$

Finally, if $\omega \leqslant v_{\kappa}$, then

$$
\begin{aligned}
i_{k-1}+ & \sum_{t \in S: t \leqslant v_{\kappa}} y_{t}+\sum_{t \in T} \gamma_{t}\left(1-z_{t}\right) \\
& \leqslant u_{k-1} \leqslant u_{k-1}+\sum_{t \in S}\left(d_{k t}+u_{t}-u_{k-1}\right) x_{t} .
\end{aligned}
$$

In the appendix we study the strength of inequalities (9) and (19) with respect to Q.

EXAMPLE 1 (continued). All facet-defining inequalities (19) of $Q$ for $[k, l]=[2,5]$ with $|S| \leqslant 1$ are

$$
\begin{aligned}
& i_{1}+12\left(1-z_{1}\right)+13\left(1-z_{2}\right)+5\left(1-z_{3}\right) \leqslant 30, \\
& i_{1}+12\left(1-z_{1}\right)+18\left(1-z_{2}\right) \leqslant 30, \\
& i_{1}+25\left(1-z_{1}\right)+5\left(1-z_{3}\right) \leqslant 30, \\
& i_{1}+30\left(1-z_{1}\right) \leqslant 30, \\
& i_{1}+18\left(1-z_{2}\right)+y_{2} \leqslant 30+29 x_{2}, \\
& i_{1}+13\left(1-z_{2}\right)+5\left(1-z_{3}\right)+y_{2} \leqslant 30+29 x_{2}, \\
& i_{1}+12\left(1-z_{1}\right)+5\left(1-z_{3}\right)+y_{3} \leqslant 30+29 x_{3}, \\
& i_{1}+25\left(1-z_{1}\right)+y_{4} \leqslant 30+29 x_{4}, \\
& i_{1}+12\left(1-z_{1}\right)+13\left(1-z_{2}\right)+y_{4} \leqslant 30+29 x_{4} .
\end{aligned}
$$

Observe that these inequalities are extensions of inequalities (5) with inventory fixed-charge variables. The first four inequalities are extensions of inequality $i_{1} \leqslant 30$, the next two are extensions of $i_{1}+y_{2} \leqslant 30+29 x_{2}$, and so on.

On the other hand, all facet-defining inequalities (9) of 2 for $[k, l]=[2,5]$ with $|S|=1$ are

$$
\begin{aligned}
& i_{1}+18\left(1-z_{2}\right)+y_{2} \leqslant 30+24 x_{2}+i_{5}, \\
& i_{1}+13\left(1-z_{2}\right)+5\left(1-z_{3}\right)+y_{2} \leqslant 30+24 x_{2}+i_{5}, \\
& i_{1}+12\left(1-z_{1}\right)+5\left(1-z_{3}\right)+y_{3} \leqslant 30+24 x_{3}+i_{5}, \\
& i_{1}+25\left(1-z_{1}\right)+y_{4} \leqslant 30+24 x_{4}+i_{5}, \\
& i_{1}+12\left(1-z_{1}\right)+13\left(1-z_{2}\right)+y_{4} \leqslant 30+24 x_{4}+i_{5} .
\end{aligned}
$$

Because the extreme point solutions of the LP relaxation have the same block structure with or without inventory fixed costs, it follows from Proposition 3 and Assumption 3 that inequalities (8), (9), and (19), which are generalizations of inequalities (1), (3), and (5), cut off all fractional extreme points of the LP relaxation of LSBI.

\subsection{Separation}

In this section, we study the separation problems for inequalities (9) and (19) and show that they can be solved in polynomial time.

THEOREM 7. There is an $O\left(n^{4}\right)$ algorithm to solve the separation problem for inequalities (9) and an $O\left(n^{3}\right)$ algorithm to solve the separation problem for inequalities (19).

Proof. Rewriting inequality (9) as

$$
\begin{aligned}
\sum_{t \in S} y_{t}+ & \sum_{t \in T} \gamma_{t}\left(1-z_{t}\right) \\
& -\sum_{t \in S} \min \left\{d_{k t}+u_{t}-u_{k-1}, d_{k l}-u_{k-1}, d_{t l}\right\} x_{t} \\
\leqslant & u_{k-1}-i_{k-1}+i_{l},
\end{aligned}
$$

for each $k$ and $l$ such that $1 \leqslant k \leqslant l \leqslant n$ and $u_{k-1}<d_{k l}$, we wish to determine sets $S \subseteq[k, l]$ and $T \subseteq[k-1, p-1]$ such that the left-hand side of (20) is maximized for a given point $(y, x, i, z)$. We formulate this problem as a longestpath problem on a directed acyclic network. The arcs on a longest path determine the sets $S$ and $T$.

Consider a directed graph $G=(V, A)$ with a single source $(k-1) \in V$ and a single sink $(l+1) \in V$. Let $t \in V$ for $t \in[k, l] \backslash\left[p+1, r^{\prime}\right]$, where $r^{\prime}=\max \left\{t \in[k, l]: u_{t}<\right.$ $\left.d_{(t+1) l}\right\}\left(r^{\prime}=k-1\right.$ if $u_{t} \geqslant d_{(t+1) l}$ for all $\left.t \in[k, p]\right)$.

There is an $\operatorname{arc}(t, j)^{a} \in A, a \in\{T, S\}$, for each $t \in V$ and $j \in V$ such that $k-1 \leqslant t<j \leqslant p-1$ so that if the path includes arc $(t, j)^{a}$, then $j$ is included in both $S$ and $T$ if $a=S, j$ is included in $T$ if $a=T(k-1$ is assumed to be in $T$ because $\{k-1\} \cup(S \cap[k, p-1]) \subseteq T \subseteq[k-1, p-1]$ for strong inequalities). Also, for $k \leqslant t \leqslant p-1<j \leqslant l+1$ with $t, j \in V, \operatorname{arc}(t, j)^{S}$ is in $A$ and if the path includes this arc, then $j \in S$. For $p \leqslant t<j \leqslant l+1$ with $t, j \in V$, $\operatorname{arc}(t, j)^{S}$ is in $A$ and if the path includes this arc, then $j \in S$. Finally, let $((k-1), p)^{S}$ be an arc in $A$; if the path includes this arc, then $p \in S$. (Note that there is no arc $(k, j)^{S}$ for $j>p$, because we must have $S \cap[k, p] \neq \varnothing$ from Condition 6 of Proposition 13.)

Now we assign lengths to the arcs. Let the length of $\operatorname{arc}(t, j)^{T}$ for $k-1 \leqslant t<j \leqslant p-1$ be

$c_{(t, j)^{T}}=d_{(t+1) j}\left(1-z_{t}\right)$.

Also, let the length of $\operatorname{arc}(t, j)^{S}$ be

$$
c_{(t, j)}=\left\{\begin{array}{cc}
d_{(t+1)(j-1)}\left(1-z_{t}\right)+y_{j}-\min \left\{d_{k t}+u_{t}-u_{k-1},\right. \\
\left.d_{k l}-u_{k-1}\right\} x_{j} \quad \text { if } k-1 \leqslant t<j \leqslant p, \\
\left(u_{k-1}-d_{k t}\right)\left(1-z_{t}\right)+y_{j}-d_{j l} x_{j} \\
\text { if } k \leqslant t<p<j \leqslant l+1, \\
y_{j}-d_{j l} x_{j} & \text { if } p \leqslant t<j \leqslant l+1,
\end{array}\right.
$$

where we use $y_{l+1}-d_{(l+1) l} x_{l+1}=0$, for simplicity of notation. It is easy to see that the total length of a path from 
Figure 4. Network for separation for inequality (9) $(k \leqslant j<t<p<v<w \leqslant l)$.

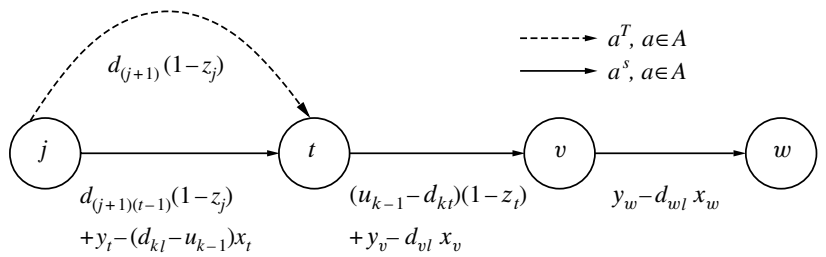

vertex $(k-1)$ to vertex $(l+1)$ equals the left-hand side of the corresponding inequality (20).

Different arc types and costs for $k \leqslant j<t<p<v<$ $w \leqslant l$ are illustrated in Figure 4. For simplicity, not all the arcs in the subgraph induced by $j, t, v, w$ are shown in the figure.

Note that $G$ is a directed acyclic graph with $O(n)$ vertices and $O\left(n^{2}\right)$ arcs. Therefore, the longest path in $G$ can be found in $O\left(n^{2}\right)$ time (see Ahuja et al. 1993). Because we solve a longest-path problem for each $k$ and $l$ such that $u_{k-1}<d_{k l}$, the overall complexity of the separation algorithm for inequalities (9) is $O\left(n^{4}\right)$. The graph $G$ can be preprocessed to remove low-length parallel arcs between two nodes, but this does not change the complexity of the algorithm.

It follows that separation for inequalities (19) is done in $O\left(n^{3}\right)$ by letting $l=n$.

Separation for inequalities (8) is done in $O(n \log n)$ similar to separation for inequalities (1).

\subsection{A Special Case: Wagner-Whitin Nonspeculative Costs}

In this section, we consider the special case of LSBI, in which the objective function satisfies the Wagner-Whitin nonspeculative property, i.e., $p_{t}+h_{t} \geqslant p_{t+1}$ for all $t \in$ $[0, n]$, where $p_{0}=p_{n+1}=0$. For this case, Pochet and Wolsey (1994) give an LP formulation for the lot-sizing problem with inventory bounds, but no inventory fixed costs. On the other hand, van Vyve and Ortega (2003) give an LP formulation for the lot sizing with inventory fixed costs, but no bounds. In this section, we generalize these results to the lot-sizing problem with inventory bounds and fixed costs by observing that the intersection of the previously given LP formulations is an integral polyhedron.

Unlike in the general cost case, if the Wagner-Whitin nonspeculative property holds, then there is an optimal inventory-minimal solution; that is, the demand in each period $t \in[1, n]$ is satisfied either from inventory or by ordering in that period, but not both, because otherwise, because the order quantity is unbounded, we can increase $y_{t}$ until $i_{t-1}=0$ without increasing the objective value.

Proposition 8. If $p_{t}+h_{t} \geqslant p_{t+1}$ for all $t \in[0, n]$, where $p_{0}=p_{n+1}=0$, then $\min \{p y+f x+h i+g z:(y, x, i, z) \in$ Q $\}$ has an optimal solution that satisfies $i_{t-1} y_{t}=0$ for all $t \in[1, n]$.
Thus, if the costs satisfy the Wagner-Whitin nonspeculative property, then there exists an optimal solution that consists of blocks of type $[k, l]_{0}^{0}$ only, which implies that, in this case, LSBI can be solved as the uncapacitated lotsizing problem by simply ignoring solutions with blocks $[k, l]_{0}^{0}$ that do not satisfy inventory upper bounds.

Next, we describe a formulation of LSBI over the inventory-minimal solutions. Note that $(y, x, i, z)$ is an inventory-minimal solution if and only if for all $t \in[1, n]$ we have $i_{t-1}=d_{t j}$ for some $j \in[t, n]$. Let $\rho_{t k}$ be a binary variable such that $\rho_{t k}=1$ if $i_{t} \geqslant d_{(t+1) k}>0$ and $\rho_{t k}=0$ otherwise for $k \in[t+1, n]$. Then, inventory-minimal solutions can be modeled as $i_{t}=\sum_{k \in[t+1, n]} d_{k} \rho_{t k}$ and $\rho_{t k}=$ $\left(1-\sum_{j \in[t+1, k]} x_{j}\right)^{+}$for $k \in\left[b_{t}, n\right]$, where $b_{t}$ is the first period after $t$ with positive demand, i.e., $b_{t}=\min \{k \in$ $\left.[t+1, n]: d_{k}>0\right\}$. Observe that the second set of equalities implies $\rho_{t k} \geqslant \rho_{t(k+1)} \geqslant \cdots \geqslant \rho_{t n}$; hence, $i_{t}=d_{(t+1) j}$ for some $j \in[t+1, n]$ (see Pochet and Wolsey 1994).

Furthermore, for an inventory-minimal solution, $i_{t} \leqslant u_{t} z_{t}$ if and only if (24) $z_{t} \geqslant \rho_{t b_{t}}$ and (23) $\sum_{j \in\left[t+1, l_{t}\right]} x_{j} \geqslant 1$, where $l_{t}$ is the first time period after $t$ in which demand cannot be completely satisfied by $i_{t}$, i.e., $l_{t}=\min \{k \in$ $\left.[t+1, n]: u_{t}<d_{(t+1) k}\right\}$. To see this, suppose that $i_{t} \leqslant u_{t} z_{t}$ holds. Then, $d_{(t+1)\left(l_{t}-1\right)} \leqslant u_{t}<d_{(t+1) l_{t}}$, and we have (23). Also, $z_{t}=0$ implies $i_{t}=0$ and from $i_{t}=\sum_{k \in[t+1, n]} d_{k} \rho_{t k}$ (inventory minimality), it follows that $\rho_{t k}=0$ for all $k \in$ $[t+1, n]$. Thus, we have (24) as well. For the other direction, suppose (23)-(24) hold. From (23), $x_{k}=1$ for some $k \in\left[t+1, l_{t}\right]$. Then, for an inventory-minimal solution, from $\rho_{t k}=\left(1-\sum_{j \in[t+1, k]} x_{j}\right)^{+}$, we have $\rho_{t k}=\cdots=\rho_{t n}=0$ and $i_{t} \leqslant d_{(t+1)(k-1)} \leqslant u_{t}$. From (24), if $z_{t}=0$, then $\rho_{t b_{t}}=0$, which, by $\rho_{t k}=\left(1-\sum_{j=t+1}^{k} x_{j}\right)^{+}$implies that $x_{j}=1$ for some $j \in\left[t+1, b_{t}\right]$. Then, again by inventory minimality, $\rho_{t b_{t}}=\cdots=\rho_{t n}=0$ and $i_{t}=0$ as $d_{(t+1) b_{t}}-1=0$.

After eliminating the order variables $y_{t}$ by substituting $d_{t}+i_{t}-i_{t-1}$, the inventory costs become $h_{t}^{\prime}=p_{t}+h_{t}-$ $p_{t+1}$ and the objective contains the constant term $K=$ $\sum_{t \in[1, n]} p_{t} d_{t}$. Because $h^{\prime} \geqslant 0$ due to the Wagner-Whitin nonspeculative costs, the problem can be stated as

$$
\begin{aligned}
& \min \sum_{t \in[1, n]} f_{t} x_{t}+\sum_{t \in[0, n]}\left(g_{t} z_{t}+h_{t}^{\prime} i_{t}\right)+K \\
& i_{t} \geqslant \sum_{k \in[t+1, n]} d_{k} \rho_{t k}, \quad t \in[0, n-1], \\
& \rho_{t k}+\sum_{j \in[t+1, k]} x_{j} \geqslant 1, \quad t \in[0, n-1], k \in\left[b_{t}, n\right], \\
& \sum_{j \in\left[t+1, l_{t}\right]} x_{j} \geqslant 1, \quad t \in[0, n-1], \\
& z_{t}-\rho_{t b_{t}} \geqslant 0, \quad t \in[0, n-1], \\
& \rho_{t k} \geqslant 0, \quad t \in[0, n-1], k \in[t+1, n], \\
& 0 \leqslant x_{t} \leqslant 1, \quad t \in[1, n], \\
& 0 \leqslant z_{t} \leqslant 1, \quad t \in[0, n], \\
& i \in \mathbb{R}_{+}^{n+1}, \quad x \in\{0,1\}^{n}, \quad z \in\{0,1\}^{n+1}, \\
& \rho \in\{0,1\}^{\left(\begin{array}{c}
n \\
(2)
\end{array}\right)}
\end{aligned}
$$


Pochet and Wolsey (1994) show that the constraint matrix for (22)-(23) and (25)-(27) is totally unimodular. Because each $z_{t}$ appears once in (24), the following statement holds.

Proposition 9. Let $w=(\rho, x, z)$ and let $A w \geqslant b$ represent constraints (22)-(27). The constraint matrix $A$ is totally unimodular.

Because the right-hand side of constraints (22)-(27) is integral and each inventory variable $i_{t}$ appears only once in constraint (21), the extreme points of

$\mathbb{Q}_{W W}=\{(x, i, z, \rho):(x, i, z, \rho)$ satisfies $(21)-(27)\}$,

are the inventory-minimal solutions of LSBI.

COROLlaRy 10. The linear program $\min \left\{f x+h^{\prime} i+g z+\right.$ $\left.K:(x, i, z, \rho) \in \mathbb{Q}_{W W}\right\}$ is an extended formulation of LSBI with Wagner-Whitin nonspeculative costs.

Projecting out the auxiliary variables $\rho$ in $\mathbb{Q}_{W W}$ and reintroducing order variables $y$, we obtain a linear programming formulation of the problem with the original variables.

THEOREM 11. LSBI with Wagner-Whitin nonspeculative costs can be formulated as the following linear program:

$\min \{p y+f x+h i+g z:(y, x, i, z)$ satisfies (29)-(35) $\}$,

where

$\sum_{j \in[t, k]}\left(y_{j}-d_{j k} x_{j}\right) \leqslant i_{k}, \quad 1 \leqslant t \leqslant k \leqslant n$,

$\sum_{j \in\left[t, l_{t-1}\right]} x_{j} \geqslant 1, \quad t \in[1, n]$,

$z_{t-1}+\sum_{j \in\left[t, b_{t-1}\right]} x_{j} \geqslant 1, \quad t \in[1, n]$,

$i_{t-1}+y_{t}=d_{t}+i_{t}, \quad t \in[1, n]$,

$0 \leqslant y_{t}, \quad t \in[1, n]$,

$0 \leqslant x_{t} \leqslant 1, \quad t \in[1, n]$,

$0 \leqslant z_{t} \leqslant 1, \quad t \in[0, n]$.

Note that inequality (29) is a special case of $(l, S)$ inequalities with $S=[k, l]$, inequality (30) is a special case of inequality (3) with $S=\left[k, l_{k}\right]$, and inequality (31) is a special case of inequality (8) with $S=\left[k, b_{k-1}\right]$.

\section{Computational Results}

To test the effectiveness of the inequalities described in $\S \S 3$ and 4 in solving LSBI, we implement a branch-andcut algorithm that incorporates these inequalities and we perform computational experiments. All computations are done on a $2 \mathrm{GHz}$ Pentium 4/Linux workstation with $1 \mathrm{~GB}$ main memory.
The data used in the experiments has the following properties: Demands are generated from integer uniform distribution between 0 and 30. Inventory upper bounds are generated from integer uniform distribution between 30 and $30(c+1)$, where $c \geqslant 0$ is a parameter to determine tightness of capacity. Order costs are generated from integer uniform distribution between 4 and 24, and holding cost is equal to 1 for every period. Let $f$ be the ratio of order fixed cost to variable inventory cost and $g$ be the ratio of order fixed cost to inventory fixed cost. To observe the effect of varying capacity and cost parameters on the computations, we let $c \in\{2,5,10,20\}, f \in\{1,000,2,000,5,000\}$, and $g \in\{2,5,10\}$, and generate five random instances for each combination.

The first set of experiments is on solving LSBI with linear inventory costs. The problem instances are solved with the MIP solver of CPLEX ${ }^{1}$ Version 8.1 Beta using first only the uncapacitated inequality (1) (Unc) and then using inequalities (1), (3), and (5) (Cap) as cutting planes in the branch-and-cut tree. Given a fractional point, we find violated inequalities (1), (3), and (5) using the separation algorithms discussed in \$3.4. CPLEX cuts are disabled in these experiments to isolate the impact due to the inequalities discussed in this paper. However, to see how CPLEX cuts would perform, we also solve the same instances with the default settings of CPLEX (Def) without adding any user cuts.

A summary of these experiments for instances with 120 and 180 time periods is reported in Tables 1 and 2. In the second column of the tables we report the average integrality gap, which is $100 \times(\mathrm{zub}-$ zinit $) / \mathrm{zub}$, where zinit is the objective value of the initial LP relaxation and zub is the objective value of the best integer solution. In the third column, we compare the average percentage improvement of the integrality gap at the root node (\% gapimp), which is $100 \times($ zroot - zinit $) /(z u b-$ zinit $)$, where zroot is the objective value of the LP at the root node after the cuts are added. Columns cuts and nodes compare the average number of cuts added, and the average number of branchand-cut nodes explored, respectively. In the last column, we report the average CPU time elapsed (in seconds) if the problem is solved within a one-hour time limit. Otherwise, we also report, in parentheses, the average percentage gap between the best lower bound and the best integer solution found in the search tree (endgap) and the number of instances that have positive end gap (unslv). Except for percentage gaps, the entries in the tables are rounded to a nearest integer.

In Tables 1 and 2, we observe that the initial integrality gap increases with the length of the planning horizon and the ratio of order fixed cost to linear inventory cost $f$. The capacitated cuts close almost all of the integrality gap for most instances and reduce the computational effort dramatically. Indeed, many instances are solved without the need for branching, and the rest require only a few branches to prove optimality. While many of the instances cannot be 
Table 1. LSBI with linear inventory holding costs, $n=120$.

\begin{tabular}{|c|c|c|c|c|c|c|c|c|c|c|c|c|c|c|}
\hline \multirow[b]{2}{*}{$f$} & \multirow[b]{2}{*}{$c$} & \multirow[b]{2}{*}{ gap } & \multicolumn{3}{|c|}{$\%$ gapimp } & \multicolumn{3}{|c|}{ cuts } & \multicolumn{3}{|c|}{ nodes } & \multicolumn{3}{|c|}{ time (endgap:unslv) } \\
\hline & & & Def & Unc & Cap & Def & Unc & Cap & Def & Unc & Cap & Def & Unc & Cap \\
\hline \multirow[t]{4}{*}{1,000} & 2 & 17.8 & 73.5 & 22.1 & 95.2 & 195 & 42 & 482 & 93,655 & $1,220,825$ & 7 & 360 & $3,574(6.9: 2)$ & 2 \\
\hline & 5 & 17.1 & 77.7 & 34.5 & 96.9 & 131 & 60 & 504 & 9,109 & 579,048 & 2 & 31 & $2,285(1.7: 2)$ & 3 \\
\hline & 10 & 17.5 & 81.1 & 51.2 & 99.6 & 97 & 81 & 529 & 13,495 & 65,678 & 1 & 37 & 335 & 2 \\
\hline & 20 & 17.8 & 83.5 & 67.4 & 99.7 & 72 & 111 & 525 & 1,666 & 2,793 & 1 & 5 & 17 & 2 \\
\hline \multirow[t]{4}{*}{2,000} & 2 & 19.6 & 60.7 & 14.3 & 95.9 & 198 & 35 & 560 & 700,111 & $1,388,269$ & 3 & $2,207(1.6: 3)$ & $3,614(10.5: 5)$ & 2 \\
\hline & 5 & 18.6 & 54.2 & 21.7 & 95.1 & 150 & 49 & 492 & 701,982 & $1,151,008$ & 6 & $1,884(0.9: 2)$ & $3,613(5.3: 5)$ & 3 \\
\hline & 10 & 18.7 & 67.0 & 36.2 & 99.6 & 108 & 73 & 533 & 308,372 & 675,722 & 1 & 867 & $2,637(2.4: 3)$ & 3 \\
\hline & 20 & 17.2 & 80.7 & 53.8 & 99.5 & 80 & 120 & 569 & 447 & 16,085 & 1 & 2 & 87 & 2 \\
\hline \multirow[t]{4}{*}{5,000} & 2 & 22.5 & 44.9 & 6.2 & 96.9 & 192 & 28 & 528 & $1,182,451$ & $1,554,172$ & 2 & $2,936(6.5: 4)$ & $3,614(15.7: 5)$ & 2 \\
\hline & 5 & 21.0 & 60.3 & 10.7 & 94.8 & 194 & 35 & 493 & 501,072 & $1,225,022$ & 3 & $1,218(1.9: 1)$ & 3,137 (9.3:4) & 3 \\
\hline & 10 & 20.9 & 53.2 & 19.3 & 100.0 & 384 & 58 & 591 & 666,193 & 875,933 & 0 & $2,184(1.7: 3)$ & $2,938(5.6: 3)$ & 3 \\
\hline & 20 & 18.0 & 68.5 & 29.9 & 100.0 & 189 & 88 & 667 & 31,493 & 166,887 & 0 & 115 & $924(0.9: 1)$ & 2 \\
\hline Average & & 18.9 & 67.1 & 28.5 & 97.8 & 166 & 65 & 539 & 350,837 & 743,454 & 2 & $988(1.1: 1)$ & $2,231(4.9: 3)$ & 2 \\
\hline
\end{tabular}

solved to optimality in an hour of CPU time with default CPLEX and with uncapacitated lot-sizing inequalities (1), when the capacitated cuts (3) and (5) are added, all of them are solved within a few seconds. Note that uncapacitated lot-sizing inequalities (1) results in small gap improvement at the root node and have high endgap on average. As expected, the root gap improvement with uncapacitated inequalities increases with maximum capacity ratio $c$.

The second set of computational experiments is on solving LSBI with linear and fixed inventory costs. In these experiments we test the marginal impact of the inequalities with fixed-charge variables (8), (9), and (19) over inequalities that do not make use of the fixed-charge variables (1), (3), and (5) as well as the marginal impact of inequalities that exploit the inventory bounds and fixed charges (9) and (19) over inequalities that only use inventory fixed charges (8). Violated inequalities (8), (9), and (19) are found as described in $\$ 4.3$. A summary of these experiments for instances with 120 and 180 time periods is reported in Tables 3 and 4. The columns labelled as Def are for default CPLEX, Unc are for runs with uncapacitated inequalities (8), Lin are for runs with inequalities (1), (3), and (5), and, finally, (Cap) are for runs with inequalities (8), (9), and (19). For these instances we set the order fixed cost to inventory fixed cost ratio $g$ equal to 10 . The initial integrality gap of the LP relaxation of LSBI with inventory fixed costs is larger than for the case with linear inventory costs only. We observe that a significant effort is spent in strengthening the LP relaxations with the cutting planes; the numbers of cuts added are in the thousands.

A comparison of column Unc and Lin with Cap show that inequalities that ignore either the capacities or the inventory fixed-charge variables are not sufficient to solve the problem efficiently. We see that the addition of inequalities (9) and (19) close almost all of the integrality gap consistently for varying order fixed-cost inventory ratios and capacities. Consequently, all of the instances that could not be solved within an hour of CPU time without inequalities (9) and (19), are solved in a few minutes when they are added. These experiments clearly demonstrate the positive impact

Table 2. LSBI with linear inventory costs, $n=180$.

\begin{tabular}{|c|c|c|c|c|c|c|c|c|c|c|c|c|c|c|}
\hline \multirow[b]{2}{*}{$f$} & \multirow[b]{2}{*}{$c$} & \multirow[b]{2}{*}{ gap } & \multicolumn{3}{|c|}{$\%$ gapimp } & \multicolumn{3}{|c|}{ cuts } & \multicolumn{3}{|c|}{ nodes } & \multicolumn{3}{|c|}{ time (endgap:unslv) } \\
\hline & & & Def & Unc & Cap & Def & Unc & Cap & Def & Unc & Cap & Def & Unc & Cap \\
\hline \multirow[t]{4}{*}{1,000} & 2 & 17.5 & 73.9 & 17.6 & 99.1 & 271 & 74 & 879 & 358,306 & 701,225 & 4 & $2,404(0.7: 2)$ & $3,608(14.2: 5)$ & 9 \\
\hline & 5 & 17.1 & 78.7 & 30.3 & 99.7 & 201 & 85 & 852 & 140,510 & 629,417 & 2 & $854(0.3: 1)$ & $3,605(8.9: 5)$ & 10 \\
\hline & 10 & 17.4 & 82.5 & 47.5 & 99.3 & 182 & 114 & 818 & 28,232 & 457,648 & 2 & 181 & $3,606(4.2: 5)$ & 9 \\
\hline & 20 & 18.5 & 77.7 & 67.6 & 99.6 & 128 & 152 & 839 & 106,858 & 112,079 & 1 & 559 & 1,400 & 12 \\
\hline \multirow[t]{4}{*}{2,000} & 2 & 19.1 & 55.1 & 10.0 & 99.8 & 230 & 58 & 972 & 620,669 & 730,623 & 1 & $2,897(6.4: 4)$ & $3,612(20.6: 5)$ & 9 \\
\hline & 5 & 18.2 & 68.7 & 18.7 & 99.7 & 186 & 72 & 821 & 397,850 & 705,143 & 1 & $1,527(2.7: 2)$ & $3,614(13.8: 5)$ & 11 \\
\hline & 10 & 18.8 & 64.8 & 32.1 & 99.7 & 190 & 100 & 881 & 402,263 & 469,990 & 1 & $1,859(1.6: 2)$ & $3,612(8.4: 5)$ & 11 \\
\hline & 20 & 19.0 & 70.0 & 51.0 & 99.9 & 169 & 158 & 738 & 175,411 & 314,706 & 0 & 1,043 & $3,589(2.5: 4)$ & 13 \\
\hline \multirow[t]{4}{*}{5,000} & 2 & 22.2 & 44.4 & 5.4 & 100.0 & 237 & 46 & 956 & 818,413 & 787,667 & 1 & $3,612(9.6: 5)$ & $3,613(24.9: 5)$ & 9 \\
\hline & 5 & 20.4 & 56.6 & 9.6 & 100.0 & 269 & 53 & 956 & 647,729 & 747,869 & 0 & $2,656(4.5: 3)$ & $3,614(17.8: 5)$ & 12 \\
\hline & 10 & 21.2 & 56.4 & 19.7 & 100.0 & 195 & 75 & 1,053 & 694,361 & 660,580 & 0 & $2,890(5.1: 4)$ & $3,613(12.4: 5)$ & 10 \\
\hline & 20 & 19.7 & 52.8 & 22.0 & 100.0 & 347 & 103 & 1,016 & 551,326 & 537,426 & 0 & $2,901(2.6: 4)$ & $3,611(8.3: 5)$ & 14 \\
\hline Average & & 19.1 & 65.1 & 27.6 & 99.7 & 217 & 91 & 898 & 411,827 & 57,119 & 1 & $1,949(2.8: 2)$ & $3,425(11.3: 5)$ & 11 \\
\hline
\end{tabular}


Table 3. LSBI with fixed and linear inventory costs, $n=120$ and $g=10$.

\begin{tabular}{|c|c|c|c|c|c|c|c|c|c|c|c|c|c|c|c|c|c|c|}
\hline \multirow[b]{2}{*}{$f$} & \multirow[b]{2}{*}{$c$} & \multirow[b]{2}{*}{ gap } & \multicolumn{4}{|c|}{$\%$ gapimp } & \multicolumn{4}{|c|}{ cuts } & \multicolumn{4}{|c|}{ nodes } & \multicolumn{4}{|c|}{ time (endgap:unslv) } \\
\hline & & & Def & Unc & Lin & Cap & Def & Unc & Lin & Cap & Def & Unc & Lin & Cap & Def & Unc & Lin & Cap \\
\hline \multirow[t]{4}{*}{1,000} & 2 & 24.5 & 82.8 & 49.6 & 67.4 & 99.5 & 373 & 1,210 & 529 & 2,395 & 116,844 & 66,587 & 758,430 & 1 & 671 & $3,615(9.4: 5)$ & $3,611(3.0: 5)$ & 51 \\
\hline & 5 & 26.0 & 88.8 & 64.0 & 65.0 & 99.8 & 325 & 1,334 & 590 & 2,376 & 55,381 & 49,587 & 652,147 & 1 & 294 & $3,617(4.8: 5)$ & $3,610(2.9: 5)$ & 57 \\
\hline & 10 & 28.1 & 88.1 & 76.6 & 64.1 & 99.9 & 288 & 1,486 & 689 & 2,531 & 192,749 & 26,742 & 548,199 & 1 & $731(0.4: 1)$ & $2,693(1.5: 2)$ & $3,610(3.7: 5)$ & 47 \\
\hline & 20 & 30.6 & 90.1 & 87.2 & 62.6 & 99.9 & 265 & 1,686 & 721 & 2,030 & 25,848 & 7,231 & 460,097 & 0 & 139 & $905(0.2: 1)$ & $3,610(4.8: 5)$ & 52 \\
\hline \multirow[t]{4}{*}{2,000} & 2 & 27.6 & 65.2 & 44.1 & 65.6 & 100.0 & 391 & 1,276 & 549 & 2,108 & 827,225 & 65,867 & 753,632 & 1 & $3,612(5.9: 5)$ & $3,614(14.8: 5)$ & $3,612(4.2: 5)$ & 54 \\
\hline & 5 & 28.9 & 81.5 & 62.0 & 61.7 & 99.6 & 345 & 1,327 & 543 & 2,438 & 447,568 & 50,818 & 675,077 & 4 & $2,206(0.4: 2)$ & $3,613(6.9: 5)$ & $3,613(4.8: 5)$ & 60 \\
\hline & 10 & 31.0 & 82.6 & 72.9 & 60.9 & 100.0 & 322 & 1,586 & 748 & 2,320 & 404,856 & 35,623 & 475,499 & 0 & $1,455(1.4: 2)$ & $2,921(3.7: 4)$ & $3,612(5.9: 5)$ & 56 \\
\hline & 20 & 33.1 & 85.8 & 85.8 & 60.7 & 99.9 & 305 & 1,812 & 921 & 2,333 & 210,006 & 9,894 & 328,056 & 1 & $961(0.2: 1)$ & 1,343 & $3,610(6.4: 5)$ & 58 \\
\hline \multirow[t]{4}{*}{5,000} & 2 & 30.8 & 65.4 & 42.9 & 64.0 & 100.0 & 433 & 1,345 & 512 & 2,552 & 900,144 & 62,652 & 789,896 & 1 & $3,610(6.8: 5)$ & $3,613(16.7: 5)$ & $3,613(6.0: 5)$ & 63 \\
\hline & 5 & 31.4 & 63.9 & 57.2 & 59.3 & 99.9 & 356 & 1,343 & 549 & 2,755 & 919,685 & 58,119 & 820,783 & 0 & $3,612(6.5: 5)$ & $3,613(10.5: 5)$ & $3,613(6.9: 5)$ & 59 \\
\hline & 10 & 34.0 & 72.3 & 65.5 & 57.0 & 100.0 & 417 & 1,484 & 641 & 2,256 & 764,752 & 46,794 & 583,127 & 0 & $2,891(4.9: 3)$ & $3,613(7.9: 5)$ & $3,611(8.6: 5)$ & 60 \\
\hline & 20 & 35.1 & 78.1 & 80.1 & 56.4 & 100.0 & 525 & 1,838 & 818 & 2,113 & 560,411 & 21,298 & 337,941 & 0 & $2,893(2.0: 4)$ & $2,680(1.9: 3)$ & $3,611(8.5: 5)$ & 57 \\
\hline Average & & 30.1 & 78.7 & 65.7 & 62.1 & 99.9 & 362 & 1,477 & 651 & 2,351 & 452,122 & 41,768 & 598,574 & 1 & $1,923(2.4: 2)$ & $2,978(6.5: 4)$ & $3,611(5.5: 5)$ & 56 \\
\hline
\end{tabular}

of inequalities (9) and (19) over uncapacitated inequalities (8) that use fixed charge variables, as well as inequalities (8), (9), and (19) over inequalities (1), (3), and (5) that do not incorporate fixed-charge variables.

Table 5 summarizes the effect of changing the order fixed cost to inventory fixed-cost ratio $g$. We observe that the smaller the ratio is, the bigger the initial integrality gap. However, although the initial gap is higher, CPLEX cuts (especially flow cover cuts) lead to a better gap improvement at the root node for the instances with small $g$. It is remarkable that even with $97 \%-98 \%$ gap improvement at the root node, thousands of branch-and-cut nodes are needed to obtain provably optimal solutions. However, for the case $g=2$ and $c=10$, default CPLEX finishes computations more quickly even when many nodes are explored. We also note that the performance of our branch-andcut algorithm is not affected significantly by the tightness of the inventory upper bounds, whereas the performance of the alternative branch-and-cut algorithms degrades with lower $c$.

Our experiments with problems that satisfy the WagnerWhitin nonspeculative cost structure show that the compact linear program in Theorem 11 is solved faster than the cutting-plane algorithm that starts with the standard formulation (LSBI). Finally, we compare the performance of a branch-and-bound algorithm that solves the strengthened formulation given by (29)-(35) (Compact) with the performance of the branch-and-cut algorithm (Cap) that uses inequalities (8), (9), and (19) on instances that do not satisfy the Wagner-Whitin nonspeculative cost structure. For this experiment, $n=180, g=10$, and order variable costs are drawn from discrete uniform distribution between 4 and 104. We increase the variance in the order variable costs so that they are not approximately nonspeculative. Recall that the strengthened formulation contains a subset of the inequalities generated by the branch-andcut algorithm. In Table 6 we report the initial integrality gap of the test problems (gap), the number of branch-andcut nodes explored, and the time to solve the problems. For the branch-and-cut algorithm, under columns rgap and cuts, we report the gap at the root node when all violated inequalities (8), (9), and (19) are added and the number of cuts added, respectively. We observe that the initial integrality gap at the root node for the compact formulation

Table 4. LSBI with fixed and linear inventory costs, $n=180$ and $g=10$.

\begin{tabular}{|c|c|c|c|c|c|c|c|c|c|c|c|c|c|c|c|c|c|c|}
\hline \multirow[b]{2}{*}{$f$} & \multirow[b]{2}{*}{$c$} & \multirow[b]{2}{*}{ gap } & \multicolumn{4}{|c|}{$\%$ gapimp } & \multicolumn{4}{|c|}{ cuts } & \multicolumn{4}{|c|}{ nodes } & \multicolumn{4}{|c|}{ time (endgap:unslv) } \\
\hline & & & Def & Unc & Lin & Cap & Def & Unc & Lin & Cap & Def & Unc & Lin & Cap & Def & Unc & Lin & Cap \\
\hline \multirow[t]{4}{*}{1,000} & 2 & 24.7 & 84.5 & 45.6 & 70.8 & 99.4 & 592 & 2,598 & 747 & 6,388 & 279,834 & 22,117 & 505,488 & 15 & $2,909(1.1: 3)$ & $3,629(13.9: 5)$ & $3,613(4.5: 5)$ & 418 \\
\hline & 5 & 26.1 & 88.9 & 56.8 & 66.2 & 99.9 & 498 & 2,778 & 885 & 7,198 & 83,519 & 20,199 & 410,016 & 1 & 753 & $3,629(10.6: 5)$ & $3,614(5.4: 5)$ & 391 \\
\hline & 10 & 28.7 & 90.1 & 69.4 & 64.3 & 99.6 & 467 & 3,074 & 940 & 7,777 & 93,670 & 16,162 & 410,060 & 3 & $926(0.0: 1)$ & $3,627(7.0: 5)$ & $3,612(6.6: 5)$ & 359 \\
\hline & 20 & 31.4 & 86.0 & 84.4 & 63.6 & 99.9 & 413 & 3,546 & 1,079 & 6,454 & 345,818 & 9,770 & 323,822 & 1 & $2,780(0.6: 2)$ & $3,624(1.9: 5)$ & $3,612(7.6: 5)$ & 410 \\
\hline \multirow[t]{4}{*}{2,000} & 2 & 27.7 & 67.8 & 41.0 & 68.3 & 99.7 & 518 & 2,851 & 680 & 4,735 & 430,593 & 21,937 & 541,781 & 4 & $2,965(7.7: 4)$ & $3,630(18.8: 5)$ & $3,613(6.1: 5)$ & 420 \\
\hline & 5 & 29.1 & 76.5 & 54.6 & 63.9 & 99.9 & 476 & 2,929 & 819 & 7,081 & 368,271 & 20,142 & 405,146 & 1 & $2,424(4.4: 3)$ & $3,628(13.3: 5)$ & $3,610(7.5: 5)$ & 433 \\
\hline & 10 & 31.9 & 75.4 & 67.8 & 61.4 & 99.9 & 441 & 3,365 & 956 & 5,653 & 466,267 & 12,844 & 336,865 & 2 & $3,117(4.7: 4)$ & $3,631(8.5: 5)$ & $3,611(8.7: 5)$ & 409 \\
\hline & 20 & 34.8 & 74.3 & 76.6 & 60.1 & 99.9 & 375 & 3,871 & 1,109 & 4,699 & 609,782 & 9,226 & 209,676 & 2 & $2,916(4.9: 4)$ & $3,632(5.8: 5)$ & $3,612(10.1: 5)$ & 468 \\
\hline \multirow[t]{4}{*}{5,000} & 2 & 31.0 & 64.6 & 41.3 & 66.7 & 99.9 & 536 & 2,891 & 732 & 5,134 & 410,827 & 20,719 & 481,684 & 2 & $2,930(10.5: 4)$ & $3,629(20.3: 5)$ & $3,614(7.6: 5)$ & 432 \\
\hline & 5 & 31.8 & 71.1 & 54.2 & 61.5 & 100.0 & 498 & 2,914 & 824 & 3,983 & 459,227 & 21,050 & 419,505 & 0 & $2,923(7.3: 4)$ & $3,629(14.7: 5)$ & $3,612(9.1: 5)$ & 389 \\
\hline & 10 & 34.5 & 71.5 & 61.9 & 57.9 & 100.0 & 386 & 3,242 & 863 & 5,768 & 562,321 & 17,503 & 385,868 & 1 & $2,898(7.2: 4)$ & $3,631(12.4: 5)$ & $3,613(10.6: 5)$ & 378 \\
\hline & 20 & 36.9 & 71.2 & 72.3 & 56.7 & 100.0 & 379 & 3,755 & 1,263 & 5,720 & 595,220 & 13,545 & 219,399 & 0 & $2,930(7.2: 4)$ & $3,655(8.4: 5)$ & $3,613(12.9: 5)$ & 437 \\
\hline Average & & 30.7 & 76.8 & 60.5 & 63.5 & 99.9 & 465 & 3,151 & 908 & 5,882 & 392,115 & 17,101 & 387,443 & 3 & $2,539(4.6: 3)$ & $3,631(11.3: 5)$ & $3,612(8.1: 5)$ & 412 \\
\hline
\end{tabular}


Table 5. LSBI with fixed and linear inventory costs, $n=180$ and $f=5000$.

\begin{tabular}{|c|c|c|c|c|c|c|c|c|c|c|c|c|c|c|}
\hline \multirow[b]{2}{*}{$g$} & \multirow[b]{2}{*}{$c$} & \multirow[b]{2}{*}{ gap } & \multicolumn{3}{|c|}{$\%$ gapimp } & \multicolumn{3}{|c|}{ cuts } & \multicolumn{3}{|c|}{ nodes } & \multicolumn{3}{|c|}{ time (endgap:unslv) } \\
\hline & & & Def & Unc & Cap & Def & Unc & Cap & Def & Unc & Cap & Def & Unc & Cap \\
\hline \multirow[t]{4}{*}{10} & 2 & 30.5 & 59.3 & 40.5 & 100.0 & 491 & 2,998 & 5,636 & 565,010 & 21,855 & 2 & $3,610(11.4: 5)$ & $3,631(20.8: 5)$ & 501 \\
\hline & 5 & 32.3 & 78.7 & 49.0 & 100.0 & 462 & 2,904 & 10,692 & 457,550 & 20,259 & 1 & $2,899(4.5: 4)$ & $3,630(17.9: 5)$ & 490 \\
\hline & 10 & 35.1 & 65.9 & 60.5 & 100.0 & 433 & 3,329 & 4,974 & 685,967 & 18,336 & 1 & $3,610(9.9: 5)$ & $3,635(13.3: 5)$ & 457 \\
\hline & 20 & 39.0 & 70.5 & 71.9 & 100.0 & 542 & 4,292 & 7,763 & 649,701 & 11,277 & 1 & $3,611(8.5: 5)$ & $3,660(9.2: 5)$ & 484 \\
\hline \multirow[t]{4}{*}{5} & 2 & 40.3 & 70.2 & 62.2 & 100.0 & 539 & 4,004 & 4,762 & 554,394 & 15,671 & 2 & $3,611(10.7: 5)$ & $3,630(15.8: 5)$ & 471 \\
\hline & 5 & 43.7 & 75.5 & 71.7 & 100.0 & 441 & 3,899 & 6,373 & 664,511 & 16,873 & 1 & $3,612(8.3: 5)$ & $3,629(12.0: 5)$ & 492 \\
\hline & 10 & 47.8 & 73.9 & 77.8 & 100.0 & 434 & 4,181 & 7,647 & 719,258 & 14,012 & 2 & $3,611(9.4: 5)$ & $3,654(9.7: 5)$ & 545 \\
\hline & 20 & 52.6 & 75.8 & 86.0 & 99.9 & 430 & 4,771 & 6,027 & 665,906 & 9,885 & 1 & $3,599(8.4: 4)$ & $3,637(5.8: 5)$ & 631 \\
\hline \multirow[t]{4}{*}{2} & 2 & 59.9 & 95.1 & 85.0 & 100.0 & 572 & 4,817 & 6,544 & 266,314 & 11,101 & 2 & $2,329(0.8: 3)$ & $3,631(8.5: 5)$ & 562 \\
\hline & 5 & 64.5 & 95.8 & 91.9 & 100.0 & 485 & 4,835 & 5,416 & 174,766 & 10,936 & 1 & $1,128(0.6: 1)$ & $3,629(4.5: 5)$ & 572 \\
\hline & 10 & 68.5 & 98.0 & 92.7 & 100.0 & 452 & 4,934 & 5,568 & 9,169 & 12,206 & 1 & 82 & $3,631(4.3: 5)$ & 602 \\
\hline & 20 & 72.5 & 97.4 & 97.3 & 100.0 & 424 & 5,282 & 8,120 & 145,534 & 8,847 & 0 & $759(0.2: 1)$ & $3,631(1.2: 5)$ & 682 \\
\hline Average & & 48.9 & 79.7 & 73.8 & 100.0 & 475 & 4,187 & 6,627 & 463,173 & 14,272 & 1 & $2,705(6.1: 4)$ & $3,636(10.3: 5)$ & 541 \\
\hline
\end{tabular}

is much lower than that of the standard formulation. However, with the addition of violated inequalities (8), (9), and (19) to the standard formulation, the root integrality gap is almost closed. Consequently, the number of nodes explored is smaller for the branch-and-cut algorithm. We observe that the tighter the inventory upper bounds, the larger the integrality gap is and the longer the solution time is for the compact formulation. Also, as the ratio of order fixed cost to variable inventory cost $(f)$ increases, the initial integrality gap of the standard formulation as well as the number of explored nodes and the elapsed time increase for the branch-and-cut algorithm. The branch-and-cut algorithm is faster than the branch-and-bound algorithm for the compact formulation for smaller $f$.

In summary, these computational experiments show that:

1. Inequalities that do not take into account inventory capacities result in a poor performance of the branch-andcut algorithm;
2. Similarly, inequalities that do not take into account inventory fixed-charge variables also result in a poor performance of the algorithm;

3. However, inequalities that exploit inventory bounds as well as fixed costs are very effective in strengthening the LP relaxations of the lot-sizing problem with inventory bounds and fixed costs consistently for a wide range of cost and capacity parameters;

4. Consequently, they are very useful in solving the problem efficiently.

\section{Concluding Remarks}

In this paper, we study the facial structure of the polyhedron of lot sizing with inventory upper bounds and fixed costs (LSBI). We first define facet-defining inequalities for the special case with linear inventory costs and then extend them to incorporate inventory fixed-charge variables. We give polynomial time separation algorithms

Table 6. LSBI with fixed and linear inventory costs, compact formulation.

\begin{tabular}{|c|c|c|c|c|c|c|c|c|c|}
\hline \multirow[b]{2}{*}{$f$} & \multirow[b]{2}{*}{$c$} & \multicolumn{3}{|c|}{ Compact } & \multicolumn{5}{|c|}{ Cap } \\
\hline & & gap & nodes & time & gap & rgap & cuts & nodes & time \\
\hline \multirow[t]{4}{*}{1,000} & 2 & 2.0 & 12,199 & 765 & 19.3 & 0.1 & 7,112 & 9 & 66 \\
\hline & 5 & 1.7 & 4,649 & 401 & 20.0 & 0.1 & 3,806 & 1 & 40 \\
\hline & 10 & 1.2 & 1,126 & 127 & 20.5 & 0.4 & 10,192 & 1 & 17 \\
\hline & 20 & 1.0 & 136 & 44 & 21.4 & 0.1 & 4,315 & 1 & 16 \\
\hline \multirow[t]{4}{*}{2,000} & 2 & 1.9 & 10,637 & 791 & 22.9 & 0.3 & 10,128 & 9 & 138 \\
\hline & 5 & 1.7 & 4,417 & 396 & 24.2 & 0.1 & 4,472 & 13 & 131 \\
\hline & 10 & 1.4 & 1,297 & 156 & 25.3 & 0.1 & 8,690 & 1 & 71 \\
\hline & 20 & 1.2 & 278 & 60 & 26.6 & 0.1 & 13,479 & 2 & 61 \\
\hline \multirow[t]{4}{*}{5,000} & 2 & 1.2 & 6,368 & 641 & 26.0 & 0.2 & 4,956 & 23 & 414 \\
\hline & 5 & 1.6 & 4,201 & 417 & 28.5 & 0.3 & 5,872 & 47 & 560 \\
\hline & 10 & 1.5 & 701 & 119 & 30.0 & 0.3 & 5,169 & 17 & 296 \\
\hline & 20 & 0.9 & 129 & 51 & 31.4 & 0.3 & 20,754 & 2 & 105 \\
\hline Average & & 1.4 & 3,845 & 331 & 24.7 & 0.2 & 8,245 & 10 & 159 \\
\hline
\end{tabular}


and a linear programming formulation of LSBI under the Wagner-Whitin nonspeculative cost structure. The computational experiments suggest that the inequalities are very effective in solving the lot-sizing problem with inventory upper bounds and fixed costs.

The inequalities described here and extensions of them may be effective in solving more complicated production/order and inventory-planning problems that contain inventory upper bounds and fixed costs as a substructure. Other classes of strong inequalities for LSBI are described in Küçükyavuz (2004). Currently we are exploring how the results for LSBI can be used for solving general capacitated fixed-charge network flow problems.

\section{Appendix. Strength of the Inequalities}

In this section, we give conditions under which the proposed inequalities are facet defining. We introduce the following notation, which will be used throughout this section: For $1 \leqslant k \leqslant l \leqslant n$ and nonempty $S \subseteq[k, l]$, let

$$
\begin{aligned}
& s=\min \{t \in S\}, \\
& s^{\prime}=\max \{t \in S\}, \\
& \hat{s}=\min \{t \in[s, l+1] \backslash S\}, \text { and } \\
& \tilde{s}=\max \{t \in S: t \leqslant p\} \quad(\tilde{s}=k-1 \text { if } S \cap[k, p]=\varnothing) .
\end{aligned}
$$

Also, let $e_{t}$ and $r_{t}$ be the unit vectors corresponding to $x_{t}$ and $y_{t}$ for $t \in[1, n]$, respectively, $q_{t}$ be the unit vector corresponding to $z_{t}$ for $t \in[0, n]$, and $r_{0}$ and $v_{n}$ be the unit vectors for $i_{0}$ and $i_{n}$. Finally, let $\epsilon>0$ be an infinitesimally small number.

Under Assumptions 1 and 2, it is easy to check that the dimension of $\mathscr{P}$ is $2 n+1$.

Proposition 12. Inequality (1) is facet defining for $\mathscr{P}$ if and only if

1. if $l<n$, then $S \neq \varnothing$,

2. $u_{s}>d_{(s+1) l}$, where $s=\min \{t \in S\}$,

3. $d_{t l}>0$ for some $t \in S$ and if $d_{t l}=0$ for $t \leqslant l$, then $t \in S$,

4. $u_{s-1}>d_{s(\hat{s}-1)}$, where $\hat{s}=\min \{t \in[s, l+1] \backslash S\}$, and

5. if $l<n$, then $u_{s^{\prime}} \geqslant d_{\left(s^{\prime}+1\right)(l+1)}$, where $s^{\prime}=\max \{t \in S\}$.

Proof (Necessity). 1. Because for $l<n, d_{l+1}\left(1-x_{l+1}\right) \leqslant i_{l}$ and $x_{l+1} \leqslant 1$ dominate inequality (1) with $S=\varnothing$, we must have $S \neq \varnothing$.

2. Assume for contradiction that $u_{s} \leqslant d_{(s+1) l}$. Then, adding the $(l, S \backslash\{s\})$ inequality, $\sum_{t \in S \backslash\{s\}} y_{t} \leqslant$ $\sum_{t \in S \backslash\{s\}} d_{t l} x_{t}+i_{l}$, and the capacity inequality $y_{s} \leqslant$ $\left(d_{s}+u_{s}\right) x_{s}$ gives an inequality at least as strong as (1) because $d_{s}+u_{s} \leqslant d_{s l}$. Therefore, from Assumption 3, we must have $u_{t}>d_{(t+1) l}$ for all $t \in[s, l-1]$.

3. If $d_{t l}=0$ for some $t \in[s, l] \backslash S$, then the $(l, S \cup\{t\})$ inequality dominates (1). If $d_{t l}=0$ for all $t \in S$, then inequality (1) is implied by the aggregated demand constraint $i_{s-1}+\sum_{t \in[s, l]} y_{t}=i_{l}$.

4. If $u_{s-1}<d_{s(\hat{s}-1)}$, then there exists a period $j \in[s+1$, $\hat{s}-1]$ such that $u_{s-1}<d_{s j}$. Then, the cut-set inequality

$\sum_{t \in[s, j]} x_{t} \geqslant 1$ is valid. Now, inequality (1),

$\sum_{t \in[s, j]} y_{t} \leqslant \sum_{t \in[s, j]} d_{t j} x_{t}+i_{j}$

and the flow balance inequality

$$
i_{j}+\sum_{t \in S \cap[j+1, l]} y_{t} \leqslant d_{(j+1) l}+i_{l},
$$

imply

$$
\begin{aligned}
\sum_{t \in S} y_{t} & \leqslant \sum_{t \in[s, j]} d_{t j} x_{t}+d_{(j+1) l}+i_{l} \\
& \leqslant \sum_{t \in[s, j]} d_{t j} x_{t}+\sum_{t \in[s, j]} d_{(j+1) l} x_{t}+i_{l} \leqslant \sum_{t \in S} d_{t l} x_{t}+i_{l},
\end{aligned}
$$

where the second-to-last inequality follows from inequality (36).

On the other hand, if $u_{s-1}=d_{s(\hat{s}-1)}$, then summing inequality (3),

$i_{s-1}+\sum_{t \in S} y_{t} \leqslant u_{s-1}+\sum_{t \in S} \min \left\{d_{s l}-u_{s-1}, d_{t l}\right\} x_{t}+i_{l}$,

inequality (1)

$\sum_{t \in[s, \hat{s}-1]} y_{t} \leqslant \sum_{t \in[s, \hat{s}-1]} d_{t(\hat{s}-1)} x_{t}+i_{\hat{s}-1}$,

and the balance equality

$i_{\hat{s}-1}+d_{s(\hat{s}-1)}=i_{s-1}+\sum_{t \in[s, \hat{s}-1]} y_{t}$,

we obtain

$\sum_{t \in S} y_{t} \leqslant \sum_{t \in S} d_{t l} x_{t}+i_{l}$

5. Because the facet induced by (1) is different from $x_{l+1}=1$, it contains a point $(x, y, i)$ such that $x_{l+1}=0$ and, by feasibility, $i_{l} \geqslant d_{l+1}$. Because this point satisfies (1) at equality, $y_{j}=d_{j(l+1)}$ for some $j \in S$, which, by feasibility, implies that $u_{s^{\prime}} \geqslant d_{\left(s^{\prime}+1\right)(l+1)}$.

(Sufficiency). To prove sufficiency, we exhibit $2 n+1$ affinely independent points on the face defined by (1). For simplicity, we represent the points in the variable space $\left(i_{0},(y, x), i_{n}\right)$. (The values of the variables $i_{t}$ for $t \in[1, n-1]$ can be obtained via the flow balance equalities.) Let $e_{t}$ and $r_{t}$ be the unit vectors corresponding to $x_{t}$ and $y_{t}$ for $t \in[1, n]$, respectively, and $r_{0}$ and $v_{n}$ be the unit vectors for $i_{0}$ and $i_{n}$. If $\hat{s} \leqslant l$, then consider the point

$$
\begin{aligned}
w_{0}= & \sum_{t \in[1, s-2]}\left(d_{t} r_{t}+e_{t}\right)+d_{(s-1)(\hat{s}-1)} r_{s-1}+e_{s-1} \\
& +d_{\hat{s} l} r_{\hat{s}}+e_{\hat{s}}+\sum_{t \in[l+1, n]}\left(d_{t} r_{t}+e_{t}\right)
\end{aligned}
$$


otherwise, consider the point

$$
\begin{aligned}
w_{0}= & \sum_{t \in[1, s-2]}\left(d_{t} r_{t}+e_{t}\right)+d_{(s-1)(\hat{s}-1)} r_{s-1}+e_{s-1} \\
& +\sum_{t \in[l+1, n]}\left(d_{t} r_{t}+e_{t}\right)
\end{aligned}
$$

on the face defined by (1). First, we exhibit $2|S|$ affinely independent points. For each $j \in S$, consider the points

$$
\begin{aligned}
& w_{j}=\left\{\begin{array}{l}
w_{0}-d_{j(\hat{s}-1)} r_{s-1}+d_{j l} r_{j}+e_{j}-d_{\hat{s} l} r_{\hat{s}}-e_{\hat{s}} \quad \text { if } j \leqslant \hat{s} \leqslant l, \\
w_{0}-d_{j(\hat{s}-1)} r_{s-1}+d_{j l} r_{j}+e_{j} \quad \text { if } j \leqslant \hat{s}=l+1, \\
w_{0}-d_{j l} r_{\hat{s}}+d_{j l} r_{j}+e_{j} \quad \text { otherwise, }
\end{array}\right. \\
& \bar{w}_{j}=w_{j}+\epsilon r_{j}+\epsilon v_{n} .
\end{aligned}
$$

Next, consider the following $2(l-s+1-|S|)$ points. For $j=\hat{s} \leqslant l$, let

$w_{j}=w_{s}+e_{\hat{s}} \quad$ and $\quad \bar{w}_{j}=w_{0}-\epsilon r_{\hat{s}}+\epsilon r_{0}$.

For each $j \in[\hat{s}+1, l] \backslash S$, consider the two points

$w_{j}=w_{0}+e_{j} \quad$ and $\quad \bar{w}_{j}=w_{j}+d_{j l} r_{j}-d_{j l} r_{\hat{s}}$.

Finally, for each $j \in[1, s-1] \cup[l+1, n]$, let

$w_{j}=\left\{\begin{array}{l}w_{s^{\prime}}-e_{j}-d_{j} r_{j}+d_{j} r_{s^{\prime}} \quad \text { if } j=l+1, \\ w_{s}-e_{j}-d_{j} r_{j}+d_{j} r_{j-1} \quad \text { otherwise, }\end{array}\right.$ and
$\bar{w}_{j}= \begin{cases}w_{0}+\epsilon r_{j}+\epsilon v_{n} \quad \text { if } j \geqslant l+1, \\ w_{0}+\epsilon r_{j}-\epsilon r_{\hat{s}} & \text { if } j \leqslant s-1 \text { and } \hat{s} \leqslant l, \\ w_{0}-\epsilon r_{j}+\epsilon r_{0} & \text { if } j=s-1 \text { and } \hat{s}=l+1, \\ w_{0}+\epsilon r_{j}-\epsilon r_{s-1} & \text { if } j \leqslant s-2 \text { and } \hat{s}=l+1 .\end{cases}$

These points are affinely independent feasible points satisfying (1) at equality (Küçükyavuz 2004).

Proposition 13. If $u_{s} \geqslant d_{(s+1) l}$, then inequality (3) is facet defining for $\mathscr{P}$ if and only if

1. $u_{k-1}<d_{k l}$;

2. $u_{\tilde{s}}>d_{(\tilde{s}+1) l}$, where $\tilde{s}=\max \{t \in S: t \leqslant p\} \quad(\tilde{s}=k-1$ if $S \cap[k, p]=\varnothing)$;

3. if $u_{s-1}<d_{s p}$, then either $S=[s, l]=[s, p]$ or $\hat{s} \in[s, p]$;

4. if $d_{t l}=0$ for some $t \in[k, l]$, then $t \in S$;

5. if $k>1$, then $u_{k-2} \geqslant d_{(k-1)(s-1)}$;

6. if $u_{k-1}=d_{k(p-1)}$, then $s<p$, else $s \leqslant p$;

7. if $l<n$, then $u_{s^{\prime}} \geqslant d_{\left(s^{\prime}+1\right)(l+1)}$; and

8. if $u_{k-1}=d_{k}+u_{k}$, then $k \in S$.

Proof. Note that, by Assumption 3, $u_{s} \geqslant d_{(s+1) l}$ if and only if $d_{k t}+u_{t} \geqslant d_{k l}$ for all $t \in S$. Then, inequality (3) simplifies as

$i_{k-1}+\sum_{t \in S} y_{t} \leqslant u_{k-1}+\sum_{t \in S} \min \left\{d_{k l}-u_{k-1}, d_{t l}\right\} x_{t}+i_{l}$.

If $u_{s}<d_{(s+1) l}$, then (3) is stronger than (37).
(Necessity). 1. If $u_{k-1}=d_{k l}$, then inequality (3) is implied by the aggregated flow balance equality

$i_{k-1}+\sum_{j \in[k, l]} y_{j}=d_{k l}+i_{l}$.

2. If $u_{\tilde{s}} \leqslant d_{(\tilde{s}+1) l}$, then inequality (3) is dominated by (5),

$i_{k-1}+\sum_{j \in S: j \leqslant p} y_{j} \leqslant u_{k-1}+\sum_{j \in S: j \leqslant p}\left(d_{k \tilde{s}}-u_{k-1}+u_{\tilde{s}}\right) x_{j}$,

and inequality (1),

$\sum_{j \in S: j>p} y_{j} \leqslant \sum_{j \in S: j>p} d_{j l} x_{j}+i_{l}$

Therefore, we must have $u_{\tilde{s}}>d_{(\tilde{s}+1) l}$. Note that, from Assumption 3, this condition implies that $u_{t}>d_{(t+1) l}$ for all $t \in[\tilde{s}, l]$.

3. Suppose that the condition is not true. Either $S=$ $[s, p]$ and $k<s \leqslant p<l$, or $[s, p] \subset S$. Because $u_{s-1}<d_{s p}$, it follows that

$\sum_{t \in[s, p]} x_{t} \geqslant 1$

Multiplying both sides of this inequality with $d_{k l}-u_{k-1}$ and adding $d_{k l}+i_{l}=i_{k-1}+\sum_{t \in[k, l]} y_{t}$, we obtain

$\sum_{t \in[k, l]} y_{t} \leqslant \sum_{t \in[s, p]}\left(d_{k l}-u_{k-1}\right) x_{t}+\left(u_{k-1}-i_{k-1}\right)+i_{l}$,

which is stronger than (3) in either case.

4. Note that $d_{t l}>0$ for all $t \in[k, p]$ because we must have $0<u_{k-1}<d_{k p}$. If $d_{t l}=0$ for some $t \in[p+1, l]$ and $t \notin S$, then inequality (3), where $S$ augmented with $t$, is stronger.

5. Because the facet defined by (3) is different from $x_{k-1}=1$, there is a point $(x, y, i)$ on the face with $x_{k-1}=0$. Because (3) is satisfied at equality, we have $i_{k-1} \geqslant d_{k(s-1)}$. As $y_{k-1}=0$, this implies that $u_{k-2} \geqslant i_{k-2} \geqslant d_{(k-1)(s-1)}$.

6. If $s>p$, then inequality (3) is obtained by adding $i_{k-1} \leqslant u_{k-1}$ and the $(l, S)$ inequality. Therefore, we must have $s \leqslant p$. However, if $u_{k-1}=d_{k(p-1)}$, then $d_{k l}-u_{k-1}=$ $d_{p l}$ and by the same argument, we need $s<p$. Note that this condition implies that $S \neq \varnothing$.

7. Follows from the same argument for Item 5 of Proposition 12.

8. Suppose that $u_{k-1}=d_{k}+u_{k}$ and $k \notin S$. Then, inequality

$i_{k}+\sum_{t \in S} y_{t} \leqslant u_{k}+\sum_{t \in S} \min \left\{d_{(k+1) l}-u_{k}, d_{t l}\right\} x_{t}+i_{l}$

is stronger than (3). This can be seen by subtracting the flow balance equality

$i_{k-1}+y_{k}=d_{k}+i_{k}$

from (3). 
(Sufficiency). We define $2 n+1$ affinely independent points on the face defined by (3). For simplicity, we represent the points in the variable space $\left(i_{0},(y, x), i_{n}\right)$. (The values of the variables $i_{t}$ for $t \in[1, n-1]$ can be obtained via the flow balance equalities.) For consistency of notation, let $d_{0}$ be zero and $e_{0}$ be the zero vector. Consider the point $w_{0}=\sum_{t \in[1, k-2]}\left(d_{t} r_{t}+e_{t}\right)+\sum_{t \in[l+1, n]}\left(d_{t} r_{t}+e_{t}\right)+\left(d_{k-1}+\right.$ $\left.u_{k-1}\right) r_{k-1}+e_{k-1}$. Note that $w_{0}$ is not feasible because $u_{k-1}<d_{k l}$. We perturb $w_{0}$ to define feasible points.

First, consider the following $2|S|$ points. For each $j \in S$, if $j \leqslant p$, then let

$w_{j}=w_{0}+e_{j}+\left(d_{k l}-u_{k-1}\right) r_{j} ;$

otherwise let

$w_{j}=\left\{\begin{array}{c}w_{0}+e_{j}+d_{j l} r_{j}+\left(d_{k(j-1)}-u_{k-1}\right) r_{\hat{s}}+e_{\hat{s}} \quad \text { if } \hat{s} \leqslant p, \\ w_{0}+e_{j}+d_{j l} r_{j}+\left(d_{k(\hat{s}-1)}-u_{k-1}\right) r_{s-1}+e_{s-1} \\ \quad+d_{\hat{s}(j-1)} r_{\hat{s}}+e_{\hat{s}} \quad \text { if } s>k, \hat{s}>p, \\ w_{0}+e_{j}+d_{j l} r_{j}+\left(d_{k(\hat{s}-1)}-u_{k-1}\right) r_{s-1}+d_{\hat{s}(j-1)} r_{\hat{s}}+e_{\hat{s}} \\ \quad \text { if } s=k, \hat{s}>p .\end{array}\right.$

Also, for $j \in S$, let

$\bar{w}_{j}= \begin{cases}w_{j}+\epsilon r_{j}+\epsilon v_{n} & \text { if } j \geqslant \tilde{s}, \\ w_{j}+\epsilon r_{j}-\epsilon r_{k-1} & \text { otherwise. }\end{cases}$

For $j=k-1$ and $k>1$, let

$$
\begin{aligned}
w_{j}= & w_{0}-e_{k-1}-\left(d_{k-1}+u_{k-1}\right) r_{k-1} \\
& +d_{(k-1)(s-1)} r_{k-2}+d_{s l} r_{s}+e_{s} \text { and } \\
\bar{w}_{j}= & w_{\tilde{s}}-\epsilon r_{k-1}+\epsilon r_{\tilde{s}} .
\end{aligned}
$$

For $j \in[k, l] \backslash S$ consider

$$
\begin{aligned}
& w_{j}=w_{s}+e_{j} \quad \text { and } \\
& \bar{w}_{j}= \begin{cases}w_{0}+\left(d_{k l}-u_{k-1}\right) r_{j}+e_{j} \\
\text { if } j \in[\hat{s}, p] \text { or } j=s-1, \hat{s}>p, \\
\bar{w}_{\hat{s}}+\epsilon r_{j}+e_{j}-\epsilon r_{\hat{s}} & \text { if } j \in[p+1, l] \backslash\{\hat{s}\}, \\
\bar{w}_{\hat{s}}+\epsilon r_{j}+e_{j}-\epsilon r_{\hat{s}} & \text { if } j \in[k, \hat{s}-1] \text { and } \hat{s} \leqslant p, \\
w_{s-1}+\epsilon r_{j}+e_{j}-\epsilon r_{s-1} & \text { if } j \in[k, s-2] \text { and } \hat{s} \geqslant p, \\
w_{0}+\left(d_{k(\hat{s}-1)}-u_{k-1}\right) r_{s-1}+e_{s-1}+d_{\hat{s} l} r_{\hat{s}}+e_{\hat{s}} & \text { if } j=\hat{s}>p \text { and } s>k, \\
w_{0}+\left(d_{k(\hat{s}-1)}-u_{k-1}\right) r_{s-1}+d_{\hat{s} l} r_{\hat{s}}+e_{\hat{s}} & \text { if } j=\hat{s}>p \text { and } s=k .\end{cases}
\end{aligned}
$$

For $j \in[l+1, n]$ and $l<n$, consider

$$
\begin{aligned}
& w_{j}=\left\{\begin{array}{ll}
w_{s^{\prime}}-e_{j}-d_{j} r_{j}+d_{j} r_{s^{\prime}} & \text { if } j=l+1, \\
w_{s^{\prime}}-e_{j}-d_{j} r_{j}+d_{j} r_{j-1} & \text { if } j \in[l+2, n],
\end{array}\right. \text { and } \\
& \bar{w}_{j}=w_{s^{\prime}}+\epsilon r_{j}+\epsilon v_{n} .
\end{aligned}
$$

For $j \in[1, k-2]$ and $k>2$, let

$w_{j}=w_{s^{\prime}}-e_{j}-d_{j} r_{j}+d_{j} r_{j-1}$ and $\bar{w}_{j}=w_{s^{\prime}}+\epsilon r_{j}-\epsilon r_{k-1}$.

Finally, let

$\bar{w}_{0}= \begin{cases}w_{s}+\epsilon r_{0}-\epsilon r_{k-1} & \text { if } k>1, \\ w_{\tilde{s}}-\epsilon r_{k-1}+\epsilon r_{\tilde{s}} & \text { if } k=1 .\end{cases}$

The $2 n+1$ points given above are affinely independent points on the face (3) (Küçükyavuz 2004).

Proposition 14. Let $S \subseteq[k, l]$ for $1 \leqslant k \leqslant l \leqslant n$. If $u_{s} \geqslant$ $d_{(s+1) l}+u_{l}$, then inequality (5) is facet defining for $\mathscr{P}$ if and only if

1. $u_{k-1}<d_{k l}+u_{l}$;

2. $S \subseteq[k, q]$;

3. if $u_{s-1}<d_{s q}$, then $S \neq[s, q]$;

4. if $k>1$, then $u_{k-2} \geqslant d_{(k-1)(s-1)}$ if $S \neq \varnothing$ and $u_{k-2} \geqslant$ $d_{k-1}+u_{k-1}$ if $S=\varnothing$; and

5. if $u_{k-1}=d_{k}+u_{k}$, then $k \in S$.

Proof. Note that by Assumption 3, $u_{s} \geqslant d_{(s+1) l}+u_{l}$ if and only if $d_{k t}+u_{t}=d_{k l}+u_{l}$ for all $t \in S$. Then, inequality (3) simplifies as

$i_{k-1}+\sum_{t \in S} y_{t} \leqslant u_{k-1}+\sum_{t \in S}\left(d_{k l}-u_{k-1}+u_{l}\right) x_{t}$.

If $u_{s}<d_{(s+1) l}+u_{l}$, then (5) is stronger than (38).

(Necessity). 1. From Assumption 3, $u_{k-1} \leqslant d_{k l}+u_{l}$. If $u_{k-1}=d_{k l}+u_{l}$, then inequality (5) is implied by the aggregated flow balance equality

$i_{k-1}+\sum_{t \in[k, l]} y_{t}=d_{k l}+i_{l} \leqslant d_{k l}+u_{l}$.

2. The statement holds trivially if $q=l$. Otherwise, from the definition of $q$, we have $d_{k l}-u_{k-1}+u_{l} \geqslant d_{(q+1) l}+u_{l} \geqslant$ $d_{t}+u_{t}$ for all $t \in[q+1, l]$. Then, if $t \in S \cap[q+1, l]$, the inequality is dominated by the one with $S^{\prime}=S \backslash\{t\}$ and $y_{t} \leqslant\left(d_{t}+u_{t}\right) x_{t}$.

3. Suppose that $u_{s-1}<d_{s q}$ and $S=[s, q]$. Then,

$\sum_{t \in[s, q]} x_{t} \geqslant 1$

is valid. Multiplying both sides of this inequality with $d_{k q}-u_{k-1}+u_{q}$, and adding $i_{q} \leqslant u_{q}$ and $i_{k-1}+\sum_{t \in[k, q]} y_{t}=$ $d_{k q}+i_{q}$, gives

$i_{k-1}+\sum_{t \in[k, q]} y_{t} \leqslant u_{k-1}+\sum_{t \in[s, q]}\left(d_{k q}-u_{k-1}+u_{q}\right) x_{t}$.

However, from Condition 2 and Assumption 3, we have $u_{q}=d_{(q+1) l}+u_{l}$. Thus, (39) equals

$i_{k-1}+\sum_{t \in[k, q]} y_{t} \leqslant u_{k-1}+\sum_{t \in[s, q]}\left(d_{k l}-u_{k-1}+u_{l}\right) x_{t}$. 
4. If $S \neq \varnothing$, the proof is the same as for Condition 5 of Proposition 13. If $S=\varnothing$ and $u_{k-2}<d_{k-1}+u_{k-1}$, then $i_{k-1} \leqslant u_{k-1}$ is dominated by the valid inequality $\left(u_{k-1}+\right.$ $\left.d_{k-1}-u_{k-2}\right)\left(1-x_{k-1}\right)+i_{k-1} \leqslant u_{k-1}$.

5. If $u_{k-1}=d_{k}+u_{k}$ and $k \notin S$, then adding inequality (5),

$i_{k}+\sum_{t \in S} y_{t} \leqslant u_{k}+\sum_{t \in S}\left(d_{(k+1) l}-u_{k}+u_{l}\right) x_{t}$,

and the flow balance equality $i_{k-1}+y_{k}=d_{k}+i_{k}$, we obtain a stronger inequality.

(Sufficiency). By Assumption 3, we have $u_{q}=d_{(q+1) l}+$ $u_{l}$, and by Condition 2 we have $S \subseteq[k, q]$ where $q \leqslant l$. Therefore, we assume below that $l=q$. We give $2 n+1$ affinely independent points on the face (5). For simplicity, we represent the points in the variable space $\left(i_{0},(y, x), i_{n}\right)$. (The values of the variables $i_{t}$ for $t \in[1, n-1]$ can be obtained via the flow balance equalities.) Let $w_{0}=$ $\sum_{j \in[1, k-1]}\left(e_{j}+d_{j} r_{j}\right)+\sum_{j \in[l+1, n]}\left(e_{j}+\bar{d}_{j} r_{j}\right)+e_{k-1}+\left(d_{k-1}+\right.$ $\left.u_{k-1}\right) r_{k-1}$, where $\bar{d}_{j}$ is the remaining demand for period $j$ when $i_{l}=u_{l}$ and there is no production in $[l, j]$, i.e., $\bar{d}_{j}=$ $d_{j}-\left(u_{l}-d_{(l+1)(j-1)}\right)^{+}$. (Let $d_{0}$ be zero and $e_{0}$ be the zero vector for $k=1$.) Note that $w_{0}$ is not feasible because $u_{k-1}<d_{k l}+u_{l}$. Similarly, let $d_{j}^{\prime}$ be the remaining demand for period $j$ when $i_{k-1}=u_{k-1}$ and there is no production in $[k, j]$, i.e., $d_{j}^{\prime}=d_{j}-\left(u_{k-1}-d_{k(j-1)}\right)^{+}$. Let $l=k$ and $s=k-1$ if $S=\varnothing$. Consider the following feasible point of $\mathscr{P}$ :

$\widetilde{w}=\left\{\begin{aligned} & w_{0}+\left(d_{k l}-u_{k-1}\right) r_{\hat{s}}+e_{\hat{s}}+\sum_{j \in[l+1, n]}\left(d_{j}-\bar{d}_{j}\right) r_{j} \\ & \text { if } S \neq \varnothing \text { and } \hat{s} \leqslant l, \\ & w_{0}+\left(d_{k l}-u_{k-1}\right) r_{s-1}+e_{s-1}+\sum_{j \in[l+1, n]}\left(d_{j}-\bar{d}_{j}\right) r_{j} \text { if } S \neq \varnothing, s>k, \hat{s}>l, \\ & w_{0}+\sum_{j \in[l+1, n]}\left(d_{j}^{\prime}-\bar{d}_{j}\right) r_{j} \text { if } S \neq \varnothing, s=k, \hat{s}>l, \\ & w_{0}+\sum_{j \in[l+1, n]}\left(d_{j}^{\prime}-\bar{d}_{j}\right) r_{j} \text { if } S=\varnothing .\end{aligned}\right.$

For $j \in S$, consider

$w_{j}=w_{0}+e_{j}+\left(d_{k l}+u_{l}-u_{k-1}\right) r_{j} \quad$ and

$\bar{w}_{j}=w_{j}+\epsilon r_{j}-\epsilon r_{k-1}$.

For $j=k-1$ and $k>1$ if $S \neq \varnothing$, then consider

$$
\begin{aligned}
w_{j}= & w_{0}-e_{k-1}-\left(d_{k-1}+u_{k-1}\right) r_{k-1}+d_{(k-1)(s-1)} r_{k-2} \\
& +\left(u_{l}+d_{s l}\right) r_{s}+e_{s},
\end{aligned}
$$

otherwise consider

$w_{j}=\widetilde{w}-e_{k-1}-\left(d_{k-1}+u_{k-1}\right) r_{k-1}+\left(d_{k-1}+u_{k-1}\right) r_{k-2}$.

Also, let

$\bar{w}_{k-1}=\widetilde{w}-\epsilon r_{j}+\epsilon r_{0}$.
For $j \in[k, l] \backslash S$, consider

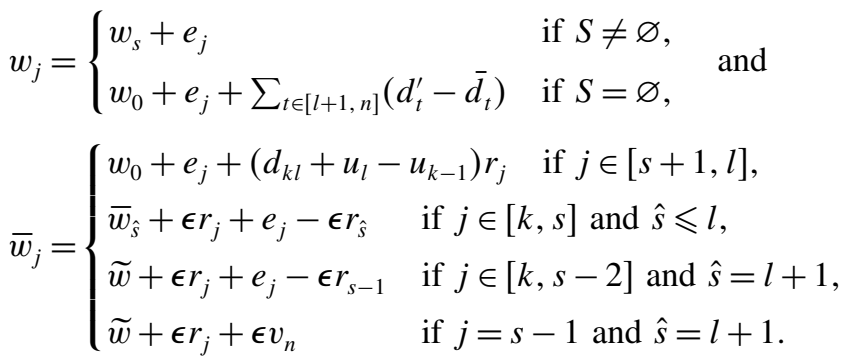

For $j \in[1, k-2]$ and $k>2$, let

$w_{j}=\widetilde{w}-e_{j}-d_{j} r_{j}+d_{j} r_{j-1} \quad$ and $\quad \bar{w}_{j}=\widetilde{w}+\epsilon r_{j}-\epsilon r_{k-1}$.

For $j=l+1$, let

$w_{j}=\left\{\begin{array}{ll}w_{s}-e_{j} & \text { if } S \neq \varnothing, \\ \bar{w}_{l}-e_{j} & \text { otherwise, }\end{array} \quad\right.$ and

$\bar{w}_{j}=\widetilde{w}+\epsilon r_{j}+\epsilon v_{n}$.

For $j \in[l+2, n]$, let $w_{j}=\widetilde{w}-e_{j}-d_{j} r_{j}+d_{j} r_{j-1}$ and $\bar{w}_{j}=$ $\widetilde{w}+\epsilon r_{j}+\epsilon v_{n}$. These $2 n+1$ points are affinely independent points satisfying inequality (5) at equality (Küçükyavuz 2004).

Under Assumptions 1 and 2, it is easy to verify that the dimension of Q is $3 n+2$.

Proposition 15. Inequality (8) is facet defining for Q under the conditions of Proposition 12 if $u_{k-1}>d_{k l}$ and either $S=\left[k, b_{k}\right]=[k, l]$ or $\left[k, b_{k}\right] \backslash S \neq \varnothing$, where $b_{k}=$ $\min \left\{t \in[k, l]: d_{t}>0\right\}$.

ProOF. For simplicity, we represent the points in the variable space $\left(i_{0},(y, x, z), i_{n}\right)$. (The values of the variables $i_{t}$ for $t \in[1, n-1]$ can be obtained via the flow balance equalities.) Let $q_{t}$ be the unit vector corresponding to $z_{t}$ for $t \in[0, n]$. For the case that $k=0$, we use $2 n+1$ points described in Proposition 12 with $q_{j}=1$ for all $j \in[0, n]$. For $j \in[0, s-2] \cup[l+1, n]$, consider $\widetilde{w}_{j}=w_{s}+$ $\sum_{t \in[0, n] \backslash\{j\}} q_{t}$. For $j \in[s-1, l]$, let $\widetilde{w}_{j}=w_{j}+\sum_{t \in[0, n] \backslash\{j\}} q_{t}$ if $j=n$. If $j<n$, then consider $\widetilde{w}_{j}=w_{j+1}+\sum_{t \in[0, n] \backslash\{j\}} q_{t}$ if $\{j+1\} \in S, \widetilde{w}_{j}=w_{0}+\sum_{t \in[0, n] \backslash\{j\}} q_{t}$ if $j+1=\hat{s}$, and $\widetilde{w}_{j}=\bar{w}_{j+1}+\sum_{t \in[0, n] \backslash\{j\}} q_{t}$ if $\{j+1\} \notin S$ and $j+1 \neq \hat{s}$. These $3 n+2$ points are affinely independent.

Now for $k>0$, let $w_{j}$ for all $j \in[1, n]$ be as described in the sufficiency proof of Proposition 12. Let $\widehat{w}_{k-1}=\sum_{t \in[1, k-2] \cup[l+1, n]}\left(d_{t} r_{t}+e_{t}\right)+d_{(k-1) l} r_{k-1}+e_{k-1}+$ $\sum_{t \in[k-1, l-1]} q_{t}$, and $\widetilde{w}_{k-1}=\widehat{w}_{k-1}+\epsilon r_{k-1}+\epsilon v_{n}+\sum_{t \in[l, n]} q_{t}$. Also, if $k>1$, let $\bar{w}_{k-1}=\widehat{w}_{k}-d_{k-1} r_{k-1}-e_{k-1}+d_{k-1} r_{k-2}+$ $q_{k-2}$. If $\left[k, b_{k}\right] \backslash S \neq \varnothing$, then let $\bar{s} \in\left[k, b_{k}\right] \backslash S$. For $j \in S$, consider the points

$\widehat{w}_{j}=\left\{\begin{array}{l}\sum_{t \in[1, k-1] \cup[l+1, n]}\left(d_{t} r_{t}+e_{t}\right)+d_{j l} r_{j}+e_{j}+\sum_{t \in[j, l-1]} q_{t} \\ \quad \text { if } j \leqslant b_{k}, \\ \sum_{t \in[1, k-1] \cup[l+1, n]}\left(d_{t} r_{t}+e_{t}\right)+d_{j l} r_{j}+e_{j}+d_{\bar{s}(j-1)} r_{\bar{s}}+e_{\bar{s}} \\ \quad \text { otherwise, }\end{array}\right.$ 
$\bar{w}_{j}=\widehat{w}_{j}+\epsilon r_{j}+\epsilon v_{n}+\sum_{t \in[l, n]} q_{t}, \quad$ and

$\widetilde{w}_{j}= \begin{cases}\widehat{w}_{j+1}+q_{j} & \text { if } j<n, \\ \widehat{w}_{j}+q_{j} & \text { otherwise. }\end{cases}$

For $j \in\left[k, b_{k}\right] \backslash S$, consider

$\widehat{w}_{j}=\sum_{t \in[1, k-1] \cup[l+1, n]}\left(d_{t} r_{t}+e_{t}\right)+d_{j l} r_{j}+e_{j}+\sum_{t \in[j, l-1]} q_{t}$,

and for $j \in\left[b_{k}+1, l\right] \backslash S$, consider

$$
\begin{aligned}
\widehat{w}_{j}= & \sum_{t \in[1, k-1] \cup[l+1, n]}\left(d_{t} r_{t}+e_{t}\right)+d_{j l} r_{j}+e_{j}+d_{\bar{s}(j-1)} r_{\bar{s}}+e_{\bar{s}} \\
& +\sum_{t \in[\bar{s}, j-2] \cup[j, l-1]} q_{t} .
\end{aligned}
$$

Also, for $j \in[k, l] \backslash S$, consider

$\bar{w}_{j}=\widehat{w}_{k-1}+e_{j}, \quad$ and

$\widetilde{w}_{j}= \begin{cases}\widehat{w}_{j+1}+q_{j} & \text { if } j<n, \\ \widehat{w}_{j}+q_{j} & \text { otherwise. }\end{cases}$

For $j \in[1, k-2] \cup[l+1, n]$, consider

$\widehat{w}_{j}= \begin{cases}\widehat{w}_{s^{\prime}}-d_{j} r_{j}-e_{j}+d_{j} r_{s^{\prime}}+q_{l} & \text { if } j=l+1, \\ \widehat{w}_{s}-d_{j} r_{j}-e_{j}+d_{j} r_{j-1}+q_{j-1} & \text { otherwise, }\end{cases}$

$\bar{w}_{j}= \begin{cases}\widehat{w}_{k-1}+\epsilon r_{j}-\epsilon r_{k-1}+\sum_{t \in[j, k-2]} q_{t} & \text { if } j \leqslant k-2, \\ \widehat{w}_{k-1}+\epsilon r_{j}+\epsilon v_{n}+\sum_{t \in[j, n]} q_{t} & \text { otherwise, }\end{cases}$

$\widetilde{w}_{j}=\widehat{w}_{k-1}+q_{j}$.

Finally, for $k>1$, consider the following two points:

$\bar{w}_{0}=\widehat{w}_{k-1}+\epsilon r_{0}-\epsilon r_{k-1}+\sum_{t \in[0, k-2]} q_{t}, \quad$ and

$\widetilde{w}_{0}=\widehat{w}_{k-1}+q_{0}$.

These $3 n+2$ points are affinely independent feasible points that satisfy (8) at equality (Küçükyavuz 2004).

Proposition 16. Inequality (9) is facet defining for $\mathbb{Q}$ if $\{k-1\} \cup([k, p-1] \cap S) \subseteq T$ and the conditions of Proposition 13 are satisfied.

Proof. For simplicity, we represent the points in the variable space $\left(i_{0},(y, x, z), i_{n}\right)$. The values of the variables $i_{t}$ for $t \in[1, n-1]$ can be obtained via the flow balance equalities. Consider the $2 n+1$ affinely independent points described in Proposition 13 with $q_{t}=1$ for all $t \in[0, n]$. For each $j \in[0, k-2] \cup[l, n]$, consider the point $\widehat{w}_{j}=w_{s}+\sum_{t \in[0, n] \backslash j\}} q_{t}$, where $w_{s}$ is as defined in Proposition 13. For each $j \in[p, l-1]$, if $\hat{s} \leqslant p$, then consider $\widehat{w}_{j}=\sum_{t \in[1, k-2] \cup[l+1, n]}\left(d_{t} r_{t}+e_{t}\right)+\left(d_{k j}-u_{k-1}\right) r_{\hat{s}}+e_{\hat{s}}+$ $\sum_{t \in[k-1, l-1] \backslash\{j\}} q_{t}+\left(d_{k-1}+u_{k-1}\right) r_{k-1}+e_{k-1}+d_{(j+1) l} r_{j+1}+$ $e_{j+1}$, otherwise let $\widehat{w}_{j}=\sum_{t \in[1, k-2] \cup[l+1, n]}\left(d_{t} r_{t}+e_{t}\right)+$ $\left(d_{k(\hat{s}-1)}-u_{k-1}\right) r_{s-1}+e_{s-1}+d_{\hat{s} j} r_{\hat{s}}+e_{\hat{s}}+\sum_{t \in[k-1, l-1] \backslash\{j\}} q_{t}+$ $\left(d_{k-1}+u_{k-1}\right) r_{k-1}+e_{k-1}+d_{(j+1) l} r_{j+1}+e_{j+1}$. For each $j \in$ $[k-1, p-1]$ with $s(j) \leqslant l$, letting $t(j)=\max \{t \in T: t \leqslant$ $j\}$, consider $\widehat{w}_{j}=\sum_{t \in[1, k-2] \cup[t(j)+1, s(j)-1] \cup[l+1, n]}\left(d_{t} r_{t}+\right.$ $\left.e_{t}\right)+\sum_{t \in[k-1, l-1] \backslash([t(j), s(j)] \cap(T \cup\{j\}))} q_{t}+d_{(k-1) t(j)} r_{k-1}+$ $e_{k-1}+d_{s(j) l} r_{s(j)}+e_{s(j)}$. For each $j \in[k-1, p-1]$ with $s(j)>l$, consider $\widehat{w}_{j}=\sum_{t \in[1, k-2]}\left(d_{t} r_{t}+e_{t}\right)+$ $d_{(k-1) t(j)} r_{k-1}+e_{k-1}+\sum_{t \in[k-1, l-1] \backslash([t(j), s(j)] \cap(T \cup\{j\}))} q_{t}+$ $\sum_{t \in[t(j)+1, s(j)-1] \cup[l+1, n]}\left(d_{t} r_{t}+e_{t}\right)$. These $3 n+2$ points are affinely independent points on the face (9) (Küçükyavuz 2004).

Proposition 17. Inequality (19) is facet defining for $\mathbb{Q}$ if $\{k-1\} \cup([k, p-1] \cap S) \subseteq T$ and the conditions of Proposition 14 are satisfied.

PROOF. For simplicity, we represent the points in the variable space $\left(i_{0},(y, x, z), i_{n}\right)$. The values of the variables $i_{t}$ for $t \in[1, n-1]$ can be obtained via the flow balance equalities. Consider the $2 n+1$ affinely independent points described in Proposition 14 with $q_{j}=1$ for all $j \in[0, n]$. For each $j \in[0, k-2]$, consider $\widehat{w}_{j}=\widetilde{w}+$ $\sum_{t \in[0, n] \backslash\{j\}} q_{t}$, where $\widetilde{w}$ is as defined in Proposition 14. For each $j \in\left[t_{\tau}, n\right]$, let $\widehat{w}_{j}=\sum_{t \in[1, k-2] \cup\left[t_{\tau}+1, n\right]}\left(d_{t} r_{t}+e_{t}\right)+$ $d_{(k-1) t_{\tau}} r_{k-1}+e_{k-1}+\sum_{t \in\left[k-1, t_{\tau}-1\right] \cup\left[t_{\tau}+1, n\right] \backslash\{j\}} q_{t}$. Finally, for $j \in\left[k-1, t_{\tau}-1\right]$, letting $t(j)=\max \{t \in T: t \leqslant j\}$, if $s(j) \leqslant l$, then consider $\widehat{w}_{j}=\sum_{t \in[1, k-2] \cup[t(j)+1, s(j)-1]}\left(d_{t} r_{t}+\right.$ $\left.e_{t}\right)+\sum_{t \in[l+1, n]}\left(\bar{d}_{t} r_{t}+e_{t}\right)+d_{(k-1) t(j)} r_{k-1}+e_{k-1}+$ $\sum_{t \in[k-1, l-1] \backslash([t(j), s(j)-1] \cap(T \cup\{j\}))} q_{t}+\left(d_{s(j) l}+u_{l}\right) r_{s(j)}+e_{s(j)}+$ $\sum_{t \in[l+1, n]} q_{t}$, where $\bar{d}_{t}$ is the effective demand in period $t$ if $i_{l}=u_{l}$. If $s(j)>l$, then consider $\widehat{w}_{j}=$ $\sum_{t \in[1, k-2] \cup[t(j)+1, s(j)-1]}\left(d_{t} r_{t}+e_{t}\right)+d_{(k-1) t(j)} r_{k-1}+e_{k-1}+$ $\sum_{t \in[k-1, l-1] \backslash([t(j), s(j)-1] \cap(T \cup\{j\}))} q_{t}+\sum_{t \in[l+1, n]}\left(d_{t} r_{t}+e_{t}\right)$. These $3 n+2$ points are affinely independent points on the face (19) (Küçükyavuz 2004).

\section{Endnote}

1. CPLEX is a trademark of ILOG, Inc.

\section{Acknowledgments}

This work was supported in part by NSF grants 0070127 and 0218265 , and a grant from ILOG, Inc. The authors are thankful to Ed Rothberg for making changes in the CPLEX MIP solver, which allowed them to add a large number of cuts efficiently and reduced the computation times significantly. The authors are also thankful to an anonymous referee for making several suggestions that improved the presentation of the paper and for noticing an error in the definition of a parameter used in the proofs in an earlier version.

\section{References}

Aggarwal, A., J. K. Park. 1993. Improved algorithms for economic lot size problems. Oper. Res. 41(3) 549-571. 
Aghezzaf, E. H., L. A. Wolsey. 1994. Modelling piecewise linear concave costs in a tree partitioning problem. Discrete Appl. Math. 50 101-109.

Agra, A., M. Constantino. 1999. Lotsizing with backlogging and start-ups: The case of Wagner-Whitin costs. Oper. Res. Lett. 25 81-88.

Ahuja, R. K., T. L. Magnanti, J. B. Orlin. 1993. Network Flows: Theory, Algorithms, and Applications. Prentice Hall, Englewood Cliffs, NJ 107-108.

Atamtürk, A. 2001. Flow pack facets of the single node fixed-charge flow polytope. Oper. Res. Lett. 29 107-114.

Atamtürk, A., D. S. Hochbaum. 2001. Capacity acquisition, subcontracting, and lot sizing. Management Sci. 47 1081-1100.

Atamtürk, A., J. C. Muñoz. 2004. A study of the lot-sizing polytope. Math. Programming 99 43-65.

Bárány, I., T. van Roy, L. A. Wolsey. 1984. Uncapacitated lot-sizing: The convex hull of solutions. Math. Programming Stud. 22 32-43.

Belvaux, G., L. A. Wolsey. 2000. bc-prod: A specialized branch-and-cut system for lot-sizing problems. Management Sci. 46 724-738.

Belvaux, G., L. A. Wolsey. 2001. Modelling practical lot-sizing problems as mixed-integer programs. Management Sci. 47(7) 993-1007.

Constantino, M. 1996. A cutting plane approach to capacitated lot-sizing with start-up costs. Math. Programming 75 353-376.

Constantino, M. 1998. Lower bounds in lot-sizing models: A polyhedral study. Math. Oper. Res. 23 101-118.

Federgruen, A., M. Tzur. 1991. A simple forward algorithm to solve general dynamic lot sizing models with $n$ periods in $O(n \log n)$ or $O(n)$ time. Management Sci. 37(2) 909-925.

Florian, M., M. Klein. 1980. Deterministic production planning with concave costs and capacity constraints. Management Sci. 26(7) 669-679.

Florian, M., J. K. Lenstra, H. G. Rinnooy Kan. 1971. Deterministic production planning: Algorithms and complexity. Management Sci. 18(1) $12-20$.

Gu, Z., G. L. Nemhauser, M. W. P. Savelsbergh. 1999. Lifted flow cover inequalities for mixed 0-1 integer programs. Math. Programming $\mathbf{8 5}$ 439-467.

Küçükyavuz, S. 2004. Polyhedral approaches to capacitated fixed-charge network flow problems. Ph.D. thesis, University of California at Berkeley, Berkeley, CA.

Leung, J. M. Y., T. L. Magnanti, R. Vachani. 1989. Facets and algorithms for capacitated lot sizing. Math. Programming 45 331-359.

Loparic, M., H. Marchand, L. A. Wolsey. 2003. Dynamic knapsack sets and capacitated lot-sizing. Math. Programming 95 53-69.

Loparic, M., Y. Pochet, L. A. Wolsey. 2001. The uncapacitated lotsizing problem with sales and safety stocks. Math. Programming 89 487-504.
Love, S. F. 1973. Bounded production and inventory models with piecewise concave costs. Management Sci. 20(3) 313-318.

Miller, A. J., G. L. Nemhauser, M. W. P. Savelsbergh. 2000. On the capacitated lot-sizing and continuous 0-1 knapsack polyhedra. Eur. J. Oper. Res. 125 298-315.

Nemhauser, G. L., L. A. Wolsey. 1988. Integer and Combinatorial Optimization. John Wiley and Sons, New York.

Ortega, F., L. A. Wolsey. 2000. A branch-and-cut algorithm for the single commodity uncapacitated fixed charge network flow problem. CORE discussion paper 2000/49, Université Catholique de Louvain, Louvain-la-Neuve, Belgium.

Padberg, M. W., T. J. van Roy, L. A. Wolsey. 1985. Valid linear inequalities for fixed charge problems. Oper. Res. 33(4) 842-861.

Pochet, Y. 1988. Valid inequalities and separation for capacitated economic lot sizing. Oper. Res. Lett. 7(3) 109-115.

Pochet, Y., L. A. Wolsey. 1988. Lot-size models with backlogging: Strong reformulations and cutting planes. Math. Programming 40 317-335.

Pochet, Y., L. A. Wolsey. 1991. Solving multi-item lot-sizing problems using strong cutting planes. Management Sci. 37 53-67.

Pochet, Y., L. A. Wolsey. 1993. Lot-sizing with constant batches: Formulation and valid inequalities. Math. Oper. Res. 18(4) 767-785.

Pochet, Y., L. A. Wolsey. 1994. Polyhedra for lot-sizing with WagnerWhitin costs. Math. Programming 67 297-323.

Stallaert, J. I. A. 1997. The complementary class of generalized flow cover inequalities. Discrete Appl. Math. 77 73-80.

Van Hoesel, C. P. M., A. P. M. Wagelmans. 1996. An $O\left(T^{3}\right)$ algorithm for the economic lot-sizing problem with constant capacities. Management Sci. 42(1) 142-150.

Van Roy, T. J., L. A. Wolsey. 1985. Valid inequalities and separation for uncapacitated fixed charge networks. Oper. Res. Lett. 4(3) 105-112.

van Vyve, M., F. Ortega. 2003. Lot-sizing with fixed charges on stocks: The convex hull. CORE discussion paper 2003/14, Université Catholique de Louvain, Louvain-la-Neuve, Belgium.

Wagelmans, A., S. van Hoesel, A. Kolen. 1992. Economic lot sizing: An $O(n \log n)$ algorithm that runs in linear time in the Wagner-Whitin case. Oper. Res. 40 S145-S156.

Wagner, H. M., T. M. Whitin. 1958. Dynamic version of the economic lot size model. Management Sci. 5 89-96.

Wolsey, L. A. 1989. Submodularity and valid inequalities in capacitated fixed charge networks. Oper. Res. Lett. 8 119-124.

Wolsey, L. A. 1998. Integer Programming. John Wiley and Sons, New York.

Wolsey, L. A. 2002. Solving multi-item lot-sizing problems with an MIP solver using classification and reformulation. Management Sci. 48(12) 1587-1602. 